$\underline{\text { Preprint typeset in JHEP style - HYPER VERSION }}$

\title{
Meson supercurrents and the Meissner effect in the Sakai-Sugimoto model
}

\author{
Anton Rebhan, Andreas Schmitt, Stefan A. Stricker \\ Institut für Theoretische Physik, Technische Universität Wien, 1040 Vienna, Austria \\ rebhana@hep.itp.tuwien.ac.at \\ aschmitt@hep.itp.tuwien.ac.at \\ stricker@hep.itp.tuwien.ac.at
}

\begin{abstract}
The Sakai-Sugimoto model provides a holographic description for chiral symmetry breaking. We use this model to investigate chirally broken phases in an external magnetic field at finite isospin and baryon chemical potentials. The equations of motion for the bulk gauge fields are solved analytically and the free energy is computed from the Yang-Mills and Chern-Simons contributions to the D8 brane action. In the case of a neutral pion condensate, a magnetic field is found to induce nonzero gradients of the Goldstone boson fields corresponding to meson supercurrents. A charged pion condensate, on the other hand, expels the magnetic field due to the Meissner effect. Upon comparing the Gibbs free energies of these two phases we find that the rotation of the chiral condensate into a charged pion condensate for finite isospin chemical potentials is partially undone by switching on a magnetic field, and we determine the critical magnetic field which removes the charged pion condensate in a first-order phase transition.
\end{abstract}

KEYWORDS: Gauge-gravity correspondence, QCD, Chiral Lagrangians. 


\section{Contents}

1. Introduction 1

2. The model 1

2.1 Geometry of confined and deconfined phases

2.2 Yang-Mills and Chern-Simons action 7

3. Equations of motion and free energy in the chirally broken phase 8

3.1 Equations of motion and ansatz including magnetic field, chemical potentials, and supercurrents

3.2 Free energy and holographic renormalization 10

4. Chirally broken phases in a magnetic field 13

4.1 Chiral rotations and resulting boundary conditions 13

4.2 Solutions of the equations of motion and free energies 16

4.2.1 Sigma phase 17

4.2.2 Pion phase and Meissner effect 20

4.3 Meson supercurrents and number densities 22

4.4 Phase diagram and critical magnetic field 26

5. Summary and outlook 31

A. General form of equations of motion 33

B. Solving the equations of motion for constant magnetic fields 34

G. Solving the equations of motion for nonconstant magnetic fields 38

D. Equations of motion and free energy in the chirally restored phase 40

\section{Introduction}

Matter in large magnetic fields occurs in nature in the interior of neutron stars [1], or can be created in the laboratory from noncentral collisions of heavy ions [2]. In both contexts it is important to understand the response of strongly-interacting quark matter, ultimately described by Quantum Chromodynamics (QCD), to an external magnetic field. And in both contexts the interplay of the magnetic field with chiral properties of the matter, in particular with chiral symmetry breaking, are crucial. While heavy-ion collisions probe the regime of the chiral phase transition at large temperatures and small chemical potentials, 
highly magnetized neutron stars ("magnetars") are composed of dense and comparably cold matter, which also may be in a phase with broken chiral symmetry. In dense quark matter, chiral symmetry can be broken by the usual chiral condensates $\langle\bar{\psi} \psi\rangle$ or, in a three-flavor system at sufficiently large densities, by diquark condensates $\langle\psi \psi\rangle$ in the color-flavor locked state [3]. In this paper we shall consider a two-flavor system at finite baryon and isospin chemical potential in the strong-coupling regime at large $N_{c}$, which may form different kinds of chiral condensates $\langle\bar{\psi} \psi\rangle$ depending on the values of temperature, the chemical potentials, and the external magnetic field.

It has been shown using a Nambu-Jona-Lasinio (NJL) model that a magnetic field can act as a catalyst for chiral symmetry breaking [4, 5], see also [6, 7]. Also chiral perturbation theory has been used to study the effect of magnetic fields [8, 9], recently for instance in the context of the deconfinement and chiral phase transitions in Refs. [10] and [11, 12], respectively. All these studies are restricted to the vacuum, i.e., they are done for the case of vanishing chemical potentials. Dense matter with nonvanishing chemical potentials in a magnetic field has been studied in the context of color superconductivity [13, 14, 15], which, due to Goldstone boson currents and the axial anomaly, can be ferromagnetic [16]. In this paper, we use the holographic model by Sakai and Sugimoto [17, 18] at nonzero isospin and baryon chemical potentials to study the effect of a magnetic field on chirally broken phases. ${ }^{1}$

Holographic models have been used extensively to study the strong-coupling limit of gauge theories since the conjecture of the AdS/CFT correspondence [22, 23, 24], i.e., the duality between (the supergravity approximation of) string theories and (the strongcoupling limit of) conformal supersymmetric gauge theories; for a review see Ref. [25]. The original and most prominent theory under investigation has been $\mathcal{N}=4$ super Yang-Mills theory which lives on the $3+1$ dimensional boundary of $\mathrm{AdS}_{5} \times \mathrm{S}^{5}$, and which is dual to type-IIB string theory living in this ten-dimensional space. While this supersymmetric gauge theory shares several properties with QCD, the differences to QCD are significant and thus it mainly serves as a model whose results should be compared with, not interpreted as, results from QCD. Therefore, a lot of effort has been spent to develop a gravity dual of QCD. Such a dual is unknown at present. Besides a "bottom-up" approach to AdS/QCD [26, 27], a promising model for capturing essential features of QCD is the Sakai-Sugimoto model, developed to study the physics of chiral symmetry breaking and meson spectra [17, 18. In contrast to the original AdS/CFT correspondence, but building upon an early proposal by Witten [28], the Sakai-Sugimoto model deals with type-IIA string theory and a five-dimensional dual gauge theory, where by means of a compactified extra dimension supersymmetry is completely broken. This extra dimension separates massless fermions of right- and left-handed chirality, which are located on the intersections of D8 and $\overline{\mathrm{D} 8}$ branes with $N_{c}$ D4 branes. As all other known gravity duals, the model is dual to the large- $N_{c}$ limit of the field theory, and the simple supergravity approximation, used in this paper as well as in most previous studies, corresponds to large values of the 't Hooft coupling.

\footnotetext{
${ }^{1}$ For effects of magnetic fields in other holographic models of strongly coupled gauge theories with flavor degrees of freedom see e.g. Refs. [19, 20, 21].
} 
With nonzero chemical potentials and a magnetic field, we shall find meson supercurrents and the Meissner effect in the chirally broken phases. Both phenomena are best understood as an analogy to (weak-coupling) superfluidity or superconductivity. For instance, a charged pion condensate of the form $\left\langle\bar{d} \gamma_{5} u\right\rangle$ can be viewed as Cooper pairing of two different fermion species, here an anti-down-quark and an up-quark. In general, Cooper pairing of two fermion species with chemical potentials $\mu_{1}$ and $\mu_{2}$ takes place at a common Fermi surface given by $\bar{\mu}=\left(\mu_{1}+\mu_{2}\right) / 2$. A mismatch in chemical potentials $\delta \mu=\left(\mu_{1}-\mu_{2}\right) / 2$ induces a "stress" on the pairing in trying to move the two Fermi surfaces apart. For not too large values of $\delta \mu$, the system can sustain the stress and the densities of the two fermion species are (at zero temperature) "locked" together, i.e., the difference in densities $\delta n=n_{1}-n_{2}$ vanishes. For larger values of $\delta \mu$, and before completely breaking the condensate, the system may respond to the stress by leaving some, but not all, fermions around the Fermi surfaces unpaired, allowing for a nonzero $\delta n$. The resulting state breaks rotational invariance, and it may even break translational invariance by giving rise to a crystalline structure. Anisotropic pion condensates in nuclear matter have been discussed a long time ago [29, 30, 31, 32]; crystalline structures of the superfluid order parameter are well-known in condensed matter physics [33, 34] as well as in dense quark matter [35], and also have been discussed in the context of chiral condensates [36], see also [37]. In either case, be it in a homogeneous manner or in a complicated crystalline structure, this unconventional pairing induces nonzero "supercurrents" in the system, see for instance Refs. [38, 39, 40]. These supercurrents are cancelled by counter-propagating currents, typically coming from unpaired fermions, such that the net current in the system vanishes.

In the case of a pion condensate of the form $\left\langle\bar{d} \gamma_{5} u\right\rangle, \bar{\mu}$ and $\delta \mu$ correspond to the isospin, $\mu_{I}$, and baryon, $\mu_{B}$, chemical potential, respectively. Consequently, one might expect anisotropic pairing upon increasing the "mismatch" $\mu_{B}$. And, corresponding to the above $\delta n$, a nonzero baryon number $n_{B}$ is expected. In the Sakai-Sugimoto model, a finite baryon number is taken into account via the Chern-Simons term. Localized baryons can be described by instantons of the effective gauge theory of the flavor branes 41, 42, 43, 44, 45, corresponding to chiral skyrmions, which in the ground state form crystals rather than a liquid [46, 47, 48]. However, we are interested in a homogeneous distribution of baryon (and isospin) density. It turns out that this can be achieved by a nonzero magnetic field in the model [49, 50, which is anyway of interest in the context of neutron star physics.

A magnetic field, however, is expelled from the charged pion condensate because a condensate of charged bosons (be it Cooper pairs or, in our case, Goldstone bosons) acts as a superconductor and thus exhibits a Meissner effect. ${ }^{2}$ Accordingly, we shall find the above expectations of a supercurrent and nonzero baryon number not realized in the charged pion condensate which remains unmodified for (not too large) magnetic fields. A meson supercurrent as well as nonzero baryon (and isospin) numbers occur, for nonzero magnetic field, instead in the phase with a neutral pion condensate. This phase is energetically preferred over the charged pion condensate beyond a critical magnetic field which we shall compute.

\footnotetext{
${ }^{2}$ Holographic models of superconductors and superfluids have recently been investigated in Refs. 51, 52, 53, 54, 55, 56], see Ref. [57] for a discussion of the Meissner effect.
} 
Our paper is organized as follows. In Sec. 2 we briefly recapitulate the Sakai-Sugimoto model. We discuss our ansatz for solutions in the presence of baryon and isospin chemical potentials and a magnetic field and derive the equations of motion and the free energy for the chirally broken phases in Sec. 3. The main part of the paper is Sec. 4 . In this part we first discuss how to incorporate different chiral condensates into the model, see Sec. 4.1. In Sec. 4.2 we solve the equations of motion for the sigma and the charged pion phase and compute their free energies. In particular, we discuss the Meissner effect in Sec. 4.2.2. The results are used to discuss the currents and number densities in these phases in Sec. 4.3. Finally, we compare their free energies to discuss the resulting phase diagram in Sec. 4.4, and we give our conclusions in Sec. 5 .

\section{The model}

In this section, the basic equations of the Sakai-Sugimoto model shall be summarized. They will be needed in the subsequent sections. For more details about the setup of the model see for instance the original papers by Sakai and Sugimoto [17, 18].

\subsection{Geometry of confined and deconfined phases}

The bulk background geometry is given by the ten-dimensional supergravity description of $N_{c}$ D4 branes in type-IIA superstring theory compactified on a circle. There are two different solutions for the metric, realized in two different temperature regimes. The transition from one to the other is interpreted as the deconfinement phase transition. Similar to the original AdS/CFT setting at finite temperature [28], the deconfined phase has a black hole which is absent in the confined phase.

The (euclidean) metric of the confined phase is given by [58]

$$
d s_{\mathrm{conf}}^{2}=\left(\frac{u}{R}\right)^{3 / 2}\left[d t^{2}+\delta_{i j} d x^{i} d x^{j}+f(u) d x_{4}^{2}\right]+\left(\frac{R}{u}\right)^{3 / 2}\left[\frac{d u^{2}}{f(u)}+u^{2} d \Omega_{4}^{2}\right] .
$$

Here, $d \Omega_{4}^{2}$ is the metric of a four-sphere, and $R$ is the curvature radius of the background which is related to the string coupling $g_{s}$ and the string length $\ell_{s}$ via

$$
R^{3}=\pi g_{s} N_{c} \ell_{s}^{3} .
$$

A crucial feature of the model is the compactified dimension $x_{4}$ which has a radius which we can parametrize through the Kaluza-Klein mass

$$
M_{\mathrm{KK}}=\frac{3}{2} \frac{u_{\mathrm{KK}}^{1 / 2}}{R^{3 / 2}}, \quad x_{4} \equiv x_{4}+2 \pi / M_{\mathrm{KK}} .
$$

This breaks supersymmetry completely by giving Kaluza-Klein masses to the adjoint fermions of the dual gauge theory and the analogue of thermal masses to the adjoint scalars, leaving only gauge bosons in the spectrum of the low-energy limit as the latter are protected by gauge symmetry [28]. The point $u=u_{\mathrm{KK}}$ is the tip of the cigar-shaped subspace spanned by $x_{4}$ and the holographic coordinate $u$, and

$$
f(u) \equiv 1-\frac{u_{\mathrm{KK}}^{3}}{u^{3}} .
$$


The subspace spanned by the euclidean time $t$ and the coordinate $u$ is cylinder-shaped, with the circumference given by the inverse temperature, $t \equiv t+1 / T$. In the deconfined phase the coordinates $t$ and $x_{4}$ interchange their roles, i.e., now the subspace spanned by $x_{4}$ and $u$ is cylinder-shaped while the subspace spanned by $t$ and $u$ is cigar-shaped. In this case, the metric is

$$
d s_{\text {deconf }}^{2}=\left(\frac{u}{R}\right)^{3 / 2}\left[\tilde{f}(u) d t^{2}+\delta_{i j} d x^{i} d x^{j}+d x_{4}^{2}\right]+\left(\frac{R}{u}\right)^{3 / 2}\left[\frac{d u^{2}}{\tilde{f}(u)}+u^{2} d \Omega_{4}^{2}\right]
$$

where temperature is related to the tip of the cigar-shaped $t$ - $u$ space $u_{T}$ via

$$
T=\frac{3}{4 \pi} \frac{u_{T}^{1 / 2}}{R^{3 / 2}}
$$

and

$$
\tilde{f}(u) \equiv 1-\frac{u_{T}^{3}}{u^{3}}
$$

The deconfinement phase transition is located at a critical temperature $T=T_{c}$ where the free energies corresponding to the two phases are identical. This occurs at $u_{\mathrm{KK}}=u_{T_{c}}$ and thus $T_{c}=M_{\mathrm{KK}} /(2 \pi)$. This critical temperature is independent of the chemical potential. Consequently, the model predicts a horizontal phase transition line in the $T$ - $\mu_{B}$ plane, in accordance with expectations from QCD at infinite number of colors $N_{c}$ [59].

The supergravity prescription depends on having the background weakly curved compared to the string scale. This is the case for large four-dimensional 't Hooft coupling 58

$$
\lambda=g_{\mathrm{YM}}^{2} N_{c}=\frac{g_{5}^{2} N_{c}}{2 \pi M_{\mathrm{KK}}^{-1}} \gg 1
$$

where the five-dimensional gauge coupling $g_{5}$ is given by $g_{5}^{2}=(2 \pi)^{2} g_{s} \ell_{s}$.

The Kaluza-Klein mass sets the energy scale below which the dual field theory is effectively four-dimensional. For large 't Hooft coupling, this scale is of the same order as the mass gap of the field theory; only for small $\lambda$, where string corrections become important, does one have duality with non-supersymmetric large- $N_{c}$ QCD in four dimensions. However, there is already ample evidence that the limit of large 't Hooft coupling, where supergravity calculations are meaningful, does provide a useful tool for unravelling certain nonperturbative features of QCD.

Sakai and Sugimoto [17] added $N_{f}$ pairs of D8 and $\overline{\mathrm{D} 8}$ branes which are transverse to the circle along $x_{4}$. The intersections of these branes with the $\mathrm{D} 4$ branes carry massless fermions in the fundamental representation of the color group of opposite chirality, which are interpreted as massless quarks of QCD. As long as $N_{f} \ll N_{c}$, the D8/ $\overline{\mathrm{D} 8}$ branes can be treated as probe branes, i.e., the backreaction on the background geometry is neglected. Below we shall take $N_{f}=2$. The $\mathrm{D} 8$ and $\overline{\mathrm{D} 8}$ branes extend in all dimensions except for the coordinate $x_{4}$ (whereas the $\mathrm{D} 4$ branes extend in the $t, x_{i}, i=1, \ldots, 4$ directions). The 
induced metrics on the probe branes in the confined and deconfined backgrounds are

$$
\begin{aligned}
d s_{\mathrm{D} 8, \mathrm{conf}}^{2} & =\left(\frac{u}{R}\right)^{3 / 2}\left(d t^{2}+\delta_{i j} d x^{i} d x^{j}\right)+\left(\frac{R}{u}\right)^{3 / 2}\left[\frac{v^{2}(u)}{f(u)} d u^{2}+u^{2} d \Omega_{4}\right], \\
d s_{\mathrm{D} 8, \mathrm{deconf}}^{2} & =\left(\frac{u}{R}\right)^{3 / 2}\left[\tilde{f}(u) d t^{2}+\delta_{i j} d x^{i} d x^{j}\right]+\left(\frac{R}{u}\right)^{3 / 2}\left[\frac{\tilde{v}^{2}(u)}{\tilde{f}(u)} d u^{2}+u^{2} d \Omega_{4}\right],
\end{aligned}
$$

where we abbreviated

$$
v(u) \equiv \sqrt{1+f^{2}(u)\left(\frac{u}{R}\right)^{3}\left(\partial_{u} x_{4}\right)^{2}}, \quad \tilde{v}(u) \equiv \sqrt{1+\left(\frac{u}{R}\right)^{3}\left(\partial_{u} x_{4}\right)^{2}} .
$$

Here the function $x_{4}(u)$ gives the embedding of the D8 branes in the $x_{4}$ - $u$ subspace.

The $\mathrm{D} 4 / \mathrm{D} 8-\overline{\mathrm{D} 8}$ setup provides the tools to study not only the deconfinement phase transition but also the chiral phase transition. In the $x_{4}$ direction, the D 8 branes are separated from the $\overline{\mathrm{D} 8}$ branes by a distance $L$. The maximal separation of the branes is $L=\pi / M_{\mathrm{KK}}$ in which case the branes are attached at opposite sides of the circle spanned by $x_{4}$. Gauge fields on the D8 and $\overline{\mathrm{D} 8}$ branes transforming under a local symmetry group $U\left(N_{f}\right)$ induce a global symmetry group $U\left(N_{f}\right)$ on the five-dimensional boundary at $u=\infty$. More precisely, a gauge symmetry on the D8 branes induces a global symmetry at the fourdimensional subspace of the holographic boundary at $x_{4}=0$, while the gauge symmetry on the $\overline{\mathrm{D} 8}$ branes induces a separate global symmetry on the four-dimensional subspace at $x_{4}=L$. Therefore the total global symmetry can be interpreted as the chiral group $U\left(N_{f}\right)_{L} \times U\left(N_{f}\right)_{R}$

So far we have viewed the gauge symmetry on the D8 branes as independent from that on the $\overline{\mathrm{D} 8}$ branes. This is correct if the branes are geometrically separate. For example in the deconfined background, where the $x_{4}-u$ subspace is cylinder-shaped, the branes follow straight lines from $u=u_{T}$ up to $u=\infty$, and thus are disconnected. However, it may also be energetically favored for the branes to be connected. In this case, the gauge symmetry reduces to joint rotations, given by the vectorial subgroup $U\left(N_{f}\right)_{L+R}$. This is exactly the symmetry breaking pattern induced by a chiral condensate (for a discussion of the chiral condensate itself as an order parameter for chiral symmetry breaking within the SakaiSugimoto model see Refs. [60, 61]). In fact, in the confined phase, where the $x_{4}$ - $u$ subspace is cigar-shaped, the branes must connect. In other words, chiral symmetry is always broken in the confined phase. Whether the branes are disconnected in the deconfined phase depends on the separation scale $L$. For sufficiently large $L$ they are always disconnected, while for smaller $L$ the connected phase may be favored for certain temperatures 62]. In other words, in the former case, deconfinement and the chiral phase transition are identical while in the latter case they differ and there exists a deconfined but chirally broken phase in the $T-\mu_{B}$ plane [63]. In this paper, we shall use maximally separated branes, i.e., $L=\pi / M_{\mathrm{KK}}$. This simplifies the treatment since in this case we always have $\partial_{u} x_{4}=0$ because the D8 branes follow geodesics. The case of not maximally separated planes, more precisely the limit where the radius of the compactified dimension is much larger than the separation distance, $1 / M_{\mathrm{KK}} \gg L$, corresponds to an NJL model on the field theory side [64]. 
Temperature and chemical potentials enter the model in very different ways. As explained above, temperature has a geometric effect on the background metric, in particular a black hole forms for sufficiently large $T$. Chemical potentials, however, enter as boundary conditions for the gauge fields on the $\mathrm{D} 8$ and $\overline{\mathrm{D} 8}$ branes, i.e., in the subsequent sections we will fix the baryon and isospin components of the temporal components of the "righthanded" and "left-handed" gauge fields at the boundary $u=\infty$ by the isospin and baryon chemical potentials. Analogously, nontrivial boundary values of the spatial components of the gauge fields have the interpretation of spatial gradients in chiral condensates, corresponding to supercurrents. We shall discuss the gauge field action associated with the flavor branes in more detail now.

\subsection{Yang-Mills and Chern-Simons action}

The total action for the $\mathrm{D} 8$ and $\overline{\mathrm{D} 8}$ branes is given by the sum of the Dirac-Born-Infeld (DBI) and the Chern-Simons actions. As indicated in the introduction and as will become clear below, the Chern-Simons term is necessary to account for nonzero baryon and isospin numbers and, in our ansatz, will be proportional to the external magnetic field. For simplicity, we shall expand the DBI action for small gauge fields such that we obtain a Yang-Mills contribution instead. This was also done for instance in Ref. [50], while other works used the full DBI action in a similar context, however for the simpler cases of a oneflavor system without isospin chemical potential 49] and without currents and magnetic field [65]. Our action takes the form

$$
S_{\mathrm{D} 8}=S_{\mathrm{YM}}+S_{\mathrm{CS}}
$$

Here, the Yang-Mills contribution is

$$
S_{\mathrm{YM}}=2 N_{f} T_{8} V_{4} \int d^{4} x d u e^{-\Phi} \sqrt{g}\left(1-\frac{\left(2 \pi \alpha^{\prime}\right)^{2}}{4 N_{f}} g^{\mu \nu} g^{\rho \sigma} \operatorname{Tr}\left[\mathcal{F}_{\nu \rho} \mathcal{F}_{\sigma \mu}\right]\right)
$$

where $T_{8}=1 /\left[(2 \pi)^{8} \ell_{s}^{9}\right]$ is the D8-brane tension, where $\alpha^{\prime}=\ell_{s}^{2}$, and where $V_{4}=8 \pi^{2} / 3$ is the volume of the unit 4-sphere. The remaining integrations are done over four-dimensional space-time $t, x_{1}, x_{2}, x_{3}$, and over the holographic coordinate $u$. In the confined (deconfined) phase the limits for this integration are $u_{\mathrm{KK}}\left(u_{T}\right)<u<\infty$, and the factor 2 on the righthand side of Eq. (2.12) accounts for integration over D8 and $\overline{\mathrm{D} 8}$ branes. In this section, all expressions are thus valid for both confined and deconfined phase, which differ, besides the integration limits for $u$, by the metric $g$. The dilaton is $e^{\Phi}=g_{s}(u / R)^{3 / 4}$, and the trace is taken over the internal $U(2)$ space (from now on $N_{f}=2$ ). Our convention for the field strength tensor is

$$
\mathcal{F}_{\mu \nu}=\partial_{\mu} \mathcal{A}_{\nu}-\partial_{\nu} \mathcal{A}_{\mu}-i\left[\mathcal{A}_{\mu}, \mathcal{A}_{\nu}\right],
$$

where $\mu, \nu=0,1,2,3, u$, and where $\mathcal{A}_{\mu}$ is the $U(2)$ gauge field. It is convenient to separate the $U(1)$ part from the gauge fields and field strengths,

$$
\mathcal{A}_{\mu}=\frac{\hat{A}_{\mu}}{2} \mathbf{1}+\frac{A_{\mu}^{a}}{2} \tau_{a}, \quad \mathcal{F}_{\mu \nu}=\frac{\hat{F}_{\mu \nu}}{2} \mathbf{1}+\frac{F_{\mu \nu}^{a}}{2} \tau_{a},
$$


where $a=1,2,3$ and $\tau_{a}$ are the Pauli matrices. With these conventions we have

$$
\hat{F}_{\mu \nu}=\partial_{\mu} \hat{A}_{\nu}-\partial_{\nu} \hat{A}_{\mu}, \quad F_{\mu \nu}^{a}=\partial_{\mu} A_{\nu}^{a}-\partial_{\nu} A_{\mu}^{a}+A_{\mu}^{b} A_{\nu}^{c} \epsilon_{a b c} .
$$

The Chern-Simons contribution in Eq. (2.11) is 42

$$
\begin{aligned}
& S_{\mathrm{CS}}=-i \frac{N_{c}}{12 \pi^{2}} \int\left\{\frac{3}{2} \hat{A} \operatorname{Tr}\left[F^{2}\right]+\frac{1}{4} \hat{A} \hat{F}^{2}+\frac{1}{2} d\left[\hat{A} \operatorname{Tr}\left(2 F A-\frac{i}{2} A^{3}\right)\right]\right\} \\
&=-i \frac{N_{c}}{96 \pi^{2}} \int d^{4} x d u\left\{\frac{3}{2} \hat{A}_{\mu}\left(F_{\nu \rho}^{a} F_{\sigma \lambda}^{a}+\frac{1}{3} \hat{F}_{\nu \rho} \hat{F}_{\sigma \lambda}\right)\right. \\
&+\left.2 \partial_{\mu}\left[\hat{A}_{\nu}\left(F_{\rho \sigma}^{a} A_{\lambda}^{a}+\frac{1}{4} \epsilon_{a b c} A_{\rho}^{a} A_{\sigma}^{b} A_{\lambda}^{c}\right)\right]\right\} \epsilon^{\mu \nu \rho \sigma \lambda},
\end{aligned}
$$

where, in the first line, we have used a notation in terms of differential forms in order to connect our expression to the one from Ref. [42] (our integration range is $u_{\mathrm{KK}}<u<\infty$; therefore, in order to integrate over D8 and $\overline{\mathrm{D} 8}$ branes we need an additional factor 2 in the prefactor compared to Eq. (2.8) in Ref. [42]). The change of numerical prefactors in going from the first to the second line comes from performing the trace and from our convention of the field strength (2.13) (the factors for the latter are hidden in the wedge products in the first line).

\section{Equations of motion and free energy in the chirally broken phase}

We can now derive the equations of motion for the gauge fields and the general form of the free energy, to be specified for various phases later. In this section and in the entire main part of the paper, we shall be concerned with the confined, i.e., chirally broken, phase whose metric $g$ is given in Eq. (2.9a). For completeness we present the equations of motion and the free energy of the deconfined, i.e., chirally restored, phase in Appendix D.

\subsection{Equations of motion and ansatz including magnetic field, chemical poten- tials, and supercurrents}

We start by taking the variation with respect to the gauge fields of the Yang-Mills and Chern-Simons Lagrangians $\mathcal{L}_{\mathrm{YM}}$ and $\mathcal{L}_{\mathrm{CS}}$. They are given by the integrands (including the prefactors outside the integral) of the actions in (2.12) and (2.16). We present the general form of the variations in Appendix $\mathrm{A}$. Here we proceed by using those general expressions for our specific ansatz.

The equations of motion obtained from the variations (A.2), (A.3), A.6), (A.7) are complicated coupled nonlinear differential equations for the gauge fields. We shall now simplify these equations by transforming the holographic coordinate $u$, by choosing a particular gauge, and by choosing a specific ansatz for the fields that captures the physics we are interested in. The new coordinate $z$ we shall use from now on is defined through

$$
u=\left(u_{\mathrm{KK}}^{3}+u_{\mathrm{KK}} z^{2}\right)^{1 / 3} .
$$

We have $z \in[-\infty, \infty]$ while $u \in\left[u_{\mathrm{KK}}, \infty\right]$. In the new coordinate, the boundaries of the connected D8 and $\overline{\mathrm{D} 8}$ branes correspond to $z=-\infty$ for $x_{4}=0$ ("left-handed fermions") 
and $z=+\infty$ for $x_{4}=L=\pi / M_{\mathrm{KK}}$ ("right-handed fermions"), while the point $z=0$ corresponds to the tip of the cigar-shaped $z-x_{4}$ subspace in the bulk. We work in a gauge where $\mathcal{A}_{z}=0$ [17, 66], see Sec. 4.1 for a discussion of this choice.

Now we specify our ansatz for the gauge fields. First, we set all components proportional to $\tau_{1}$ and $\tau_{2}$ in flavor space to zero and may then, for notational convenience drop the superscript 3 from the gauge fields and field strengths. Consequently, in the following we only have gauge fields and field strengths with a hat $(\hat{A}, \hat{F})$, corresponding to the 1-components, and without any flavor index $(A, F)$, corresponding to the $\tau_{3}$-components. This choice simplifies the calculations significantly but is a restriction for the possible chiral condensates we can capture, as we shall explain in Sec. 4.1.

The magnetic field is introduced as follows. The electromagnetic gauge group with generator $Q=\operatorname{diag}\left(q_{1}, q_{2}\right)$, where $q_{1}$ and $q_{2}$ are the electric charges of the quark flavors, is a subgroup of $U(2)_{L} \times U(2)_{R}$. The magnetic field $\mathcal{B}_{\mathrm{em}}$ thus has baryon and isospin components, $Q \mathcal{B}_{\mathrm{em}}=\hat{\mathcal{B}} \mathbf{1}+\mathcal{B} \tau_{3}$, or

$$
\hat{\mathcal{B}}=\frac{q_{1}+q_{2}}{2} \mathcal{B}_{\mathrm{em}}, \quad \mathcal{B}=\frac{q_{1}-q_{2}}{2} \mathcal{B}_{\mathrm{em}}
$$

We are interested in a system of up and down flavors, i.e., $q_{1}=2 / 3 e, q_{2}=-1 / 3 e$ with $e^{2}=4 \pi / 137$ and $\hat{\mathcal{B}}=e \mathcal{B}_{\mathrm{em}} / 6, \mathcal{B}=e \mathcal{B}_{\mathrm{em}} / 2$, but mostly we shall derive general results, keeping $\hat{\mathcal{B}}$ and $\mathcal{B}$ independent of each other. We should recall that the gauge symmetry in the bulk corresponds to a global symmetry at the boundary. Therefore, there is no electromagnetic gauge symmetry at the boundary, and in this sense $\mathcal{B}_{\mathrm{em}}$ is not a dynamical magnetic field.

We consider a spatially homogeneous magnetic field and, without loss of generality, let it point into the 3 -direction. This requires nonzero field strengths $\hat{F}_{12}$ and $F_{12}$. We can therefore choose the ansatz

$$
\begin{array}{ll}
\hat{A}_{1}(\mathbf{x}, z)=-x_{2} \frac{\hat{b}(z)}{2}, & \hat{A}_{2}(\mathbf{x}, z)=x_{1} \frac{\hat{b}(z)}{2} \\
A_{1}(\mathbf{x}, z)=-x_{2} \frac{b(z)}{2}, & A_{2}(\mathbf{x}, z)=x_{1} \frac{b(z)}{2},
\end{array}
$$

such that $\hat{F}_{12}(z)=\hat{b}(z), F_{12}(z)=b(z)$, and the boundary values at $z= \pm \infty$ of $\hat{b}(z), b(z)$ given by $\hat{\mathcal{B}}, \mathcal{B}$. (Note that for non-constant $\hat{b}(z), b(z)$, we also have nonzero field strengths $\left.\hat{F}_{i z}, F_{i z} \cdot\right)$

Next we account for the chemical potentials. This is done by relating the boundary values at $z= \pm \infty$ for the gauge fields $\hat{A}_{0}(z)$ and $A_{0}(z)$ with the baryon and isospin chemical potentials $\mu_{B}$ and $\mu_{I}$ 46, 63, 65. Consequently, we may have nonzero field strengths $\hat{F}_{0 z}$, $F_{0 z}$. It turns out that within this ansatz nonzero values of the spatial gauge fields may be induced, i.e., we have to take into account $\hat{A}_{3}(z), A_{3}(z)$ and thus the field strengths $\hat{F}_{3 z}, F_{3 z}$. The boundary values at $z= \pm \infty$ of the spatial gauge fields are identified with the gradients of the meson fields [17, 49]. These gradients correspond, according to the usual hydrodynamic theory of a superfluid [67, 68], to "supercurrents", i.e., currents of the condensate, in our context for instance the current of a pion condensate; see also Refs. [38, 39]. Consequently, we shall identify $\hat{A}_{3}( \pm \infty), A_{3}( \pm \infty)$ with meson supercurrents $\hat{\jmath}$, 
J. The supercurrents are not external parameters, hence we shall minimize the free energy with respect to them [49, 50]. They should not be confused with the "normal" currents $\mathcal{J}_{i}=\delta S_{\text {eff }} / \delta A^{i}$, discussed in the Sakai-Sugimoto model in detail in Refs. [69, 43, and computed below in Sec. 4.2.1. The supercurrents rather act as a source for the normal currents.

Now we can insert the ansatz and the coordinate transformation (3.1) into the general equations of motion (A.2), (A.3), (A.6), (A.7). We then need to replace $\mathcal{A}_{0} \rightarrow i \mathcal{A}_{0}$, since we are working in euclidean space. We find the following equations for the magnetic field

$$
\partial_{z}\left[k(z) \partial_{z} \hat{b}\right]=\partial_{z}\left[k(z) \partial_{z} b\right]=0
$$

where

$$
k(z) \equiv u_{\mathrm{KK}}^{3}+u_{\mathrm{KK}} z^{2} .
$$

They arise from the Yang-Mills variation with respect to the spatial gauge field, Eqs. (A.2b) and (A.3b) and contain no contribution from the Chern-Simons term. Moreover, they decouple from the equations for the other fields, which are

$$
\begin{aligned}
& \partial_{z}\left[k(z) \hat{F}_{z 0}\right]=\frac{\alpha u_{\mathrm{KK}}^{2}}{M_{\mathrm{KK}}^{2}}\left[b(z) F_{z 3}+\hat{b}(z) \hat{F}_{z 3}\right], \\
& \partial_{z}\left[k(z) F_{z 0}\right]=\frac{\alpha u_{\mathrm{KK}}^{2}}{M_{\mathrm{KK}}^{2}}\left[b(z) \hat{F}_{z 3}+\hat{b}(z) F_{z 3}\right], \\
& \partial_{z}\left[k(z) \hat{F}_{z 3}\right]=\frac{\alpha u_{\mathrm{KK}}^{2}}{M_{\mathrm{KK}}^{2}}\left[b(z) F_{z 0}+\hat{b}(z) \hat{F}_{z 0}\right], \\
& \partial_{z}\left[k(z) F_{z 3}\right]=\frac{\alpha u_{\mathrm{KK}}^{2}}{M_{\mathrm{KK}}^{2}}\left[b(z) \hat{F}_{z 0}+\hat{b}(z) F_{z 0}\right],
\end{aligned}
$$

where

$$
\alpha \equiv \frac{27 \pi}{2 \lambda}
$$

In all four equations in (3.6) the left-hand side comes from the variation of the Yang-Mills contribution, while the right-hand side originates from the Chern-Simons contribution. We

see that the latter is proportional to the magnetic field. The equations (3.4) and (3.6) shall be solved analytically in Sec. 4 . Before doing so we use these equations to derive a simple expression for the free energy.

\subsection{Free energy and holographic renormalization}

With the metric of the confined phase (2.1), the relations between the parameters of the model (2.2), (2.3), (2.8), and the new coordinate $z$ (3.1), the Yang-Mills part of the action (2.12) can be written as

$$
S_{\mathrm{YM}}=\kappa \int d^{4} x \int_{-\infty}^{\infty} d z\left\{\frac{16 M_{\mathrm{KK}}^{2} k^{2 / 3}(z)}{9\left(2 \pi \alpha^{\prime}\right)^{2} u_{\mathrm{KK}}}+\frac{M_{\mathrm{KK}}^{2}}{u_{\mathrm{KK}}^{2}} k(z) \operatorname{Tr}\left[\mathcal{F}_{z \mu}^{2}\right]+\frac{1}{2} h(z) \operatorname{Tr}\left[\mathcal{F}_{\mu \nu}^{2}\right]\right\}
$$

where $\mu, \nu=0,1,2,3$. Here, $k(z)$ is given in Eq. (3.5),

$$
h(z) \equiv\left(u_{\mathrm{KK}}^{3}+u_{\mathrm{KK}} z^{2}\right)^{-1 / 3},
$$


and

$$
\kappa \equiv \frac{\lambda N_{c}}{216 \pi^{3}} .
$$

In deriving Eq. (3.8) we have used that the field strengths are symmetric or antisymmetric functions of $z$. We shall see later that this is indeed the case for all phases we consider. This form of the action is general, and it is straightforward to insert our ansatz discussed in the previous subsection.

To compute the Chern-Simons contribution to the free energy we first note that the surface term (last term on the right-hand side of Eq. (2.16)) gives a nonzero contribution. Within our ansatz the term $\propto d\left(\hat{A} \operatorname{Tr}\left[A^{3}\right]\right)$ vanishes since our only nonzero flavor components of the gauge fields are proportional to 1 and $\tau_{3}$; however, the term $\propto d(\hat{A} \operatorname{Tr}[F A])$ does not vanish. We find

$$
\begin{aligned}
\hat{A}_{\mu} F_{\nu \rho} F_{\sigma \lambda} \epsilon^{\mu \nu \rho \sigma \lambda}= & 8 b\left(\hat{A}_{3} F_{z 0}-\hat{A}_{0} F_{z 3}\right) \\
\hat{A}_{\mu} \hat{F}_{\nu \rho} \hat{F}_{\sigma \lambda} \epsilon^{\mu \nu \rho \sigma \lambda}= & 8 \hat{b}\left(\hat{A}_{3} \hat{F}_{z 0}-\hat{A}_{0} \hat{F}_{z 3}\right) \\
\partial_{\mu}\left(\hat{A}_{\nu} F_{\rho \sigma} A_{\lambda}\right) \epsilon^{\mu \nu \rho \sigma \lambda}= & 2 b\left(A_{3} \hat{F}_{z 0}-A_{0} \hat{F}_{z 3}+2 \hat{A}_{0} F_{z 3}-2 \hat{A}_{3} F_{z 0}\right) \\
& +2 \hat{b}\left(A_{3} F_{z 0}-A_{0} F_{z 3}\right) .
\end{aligned}
$$

Inserting these expressions into the Chern-Simons action (2.16) yields, with $\mathcal{A}_{0} \rightarrow i \mathcal{A}_{0}$ and $N_{c} /\left(16 \pi^{2}\right)=\alpha \kappa$,

$$
\begin{aligned}
& S_{\mathrm{CS}}=\frac{\alpha \kappa}{3} \int d x^{4} \int_{-\infty}^{\infty} d z {\left[\hat{b}\left(\hat{A}_{3} \hat{F}_{z 0}+A_{3} F_{z 0}-\hat{A}_{0} \hat{F}_{z 3}-A_{0} F_{z 3}\right)\right.} \\
&\left.+b\left(\hat{A}_{3} F_{z 0}+A_{3} \hat{F}_{z 0}-\hat{A}_{0} F_{z 3}-A_{0} \hat{F}_{z 3}\right)\right] \\
&=\frac{\kappa M_{\mathrm{KK}}^{2}}{3 u_{\mathrm{KK}}^{2}} \frac{V}{T}\left\{\int_{-\infty}^{\infty} d z k(z)\left(\hat{F}_{z 0}^{2}+F_{z 0}^{2}-\hat{F}_{z 3}^{2}-F_{z 3}^{2}\right)\right. \\
&\left.-\left[k(z)\left(\hat{A}_{0} \hat{F}_{z 0}+A_{0} F_{z 0}-\hat{A}_{3} \hat{F}_{z 3}-A_{3} F_{z 3}\right)\right]_{z=-\infty}^{z=+\infty}\right\},
\end{aligned}
$$

where, in the second step, we have used the equations of motion (3.6), and where $V$ is the three-dimensional volume of space and $T$ the temperature. In changing the integration over the holographic coordinate from $u \in\left[u_{\mathrm{KK}}, \infty\right]$ to $z \in[-\infty, \infty]$ we have assumed that the integrand is symmetric in $z$. In all phases we consider this turns out to be the case. Putting the Yang-Mills and Chern-Simons contribution together, we obtain the free energy density $\Omega \equiv T\left(S_{\mathrm{YM}}+S_{\mathrm{CS}}\right) / V$

$$
\begin{aligned}
\Omega= & \Omega_{g}+\Omega_{b}+\frac{\kappa M_{\mathrm{KK}}^{2}}{6 u_{\mathrm{KK}}^{2}} \int_{-\infty}^{\infty} d z k(z)\left(-\hat{F}_{z 0}^{2}-F_{z 0}^{2}+\hat{F}_{z 3}^{2}+F_{z 3}^{2}\right) \\
& -\frac{\kappa M_{\mathrm{KK}}^{2}}{3 u_{\mathrm{KK}}^{2}}\left[k(z)\left(\hat{A}_{0} \hat{F}_{z 0}+A_{0} F_{z 0}-\hat{A}_{3} \hat{F}_{z 3}-A_{3} F_{z 3}\right)\right]_{z=-\infty}^{z=+\infty},
\end{aligned}
$$

where the geometric contribution $\Omega_{g}$ is given by the field-independent first term on the right-hand side of Eq. (3.8). This term is independent of all gauge fields and field strengths and thus plays no role in discussing the physical properties of a given phase. Moreover, we 
shall only compare free energies of phases with identical embedding of the flavor branes. Hence, for our purpose, this term can simply be dropped from now on. The term $\Omega_{b}$ in Eq. (3.13) is given by

$$
\begin{aligned}
\Omega_{b} \equiv & \frac{\kappa}{2} \int_{-\infty}^{\infty} d z h(z)\left[\hat{b}^{2}(z)+b^{2}(z)\right] \\
& +\frac{\kappa M_{\mathrm{KK}}^{2}}{4 u_{\mathrm{KK}}^{2}} \frac{\int d x_{1} d x_{2}\left(x_{1}^{2}+x_{2}^{2}\right)}{\int d x_{1} d x_{2}} \int_{-\infty}^{\infty} d z k(z)\left[\left(\partial_{z} \hat{b}\right)^{2}+\left(\partial_{z} b\right)^{2}\right] .
\end{aligned}
$$

Both contributions of $\Omega_{b}$ solely depend on the magnetic field (remember that the equations of motion for $\hat{b}$ and $b$ (3.4) decouple from the other field equations). Therefore, $\Omega_{b}$ is irrelevant for minimizing the free energy with respect to the supercurrents $\hat{\jmath}$ and $\jmath$. However, it can play a role when comparing free energies. This poses a problem, as both terms of $\Omega_{b}$ are divergent.

Let us first consider only constant functions $b(z)=\mathcal{B}$ and $\hat{b}(z)=\hat{\mathcal{B}}$, for which only the first term in $\Omega_{b}$ is present. Since we have already divided by the volume $V$ of 3 -space, we would expect a finite energy density from a homogeneous magnetic field, but because of the extra holographic dimension, this is not the case. In fact, since $h(z) \sim z^{-2 / 3}$, the divergence of $\Omega_{b}$ comes from the $|z| \rightarrow \infty$ limits of integration and is thus a typical holographic divergence which can be treated by holographic renormalization [70. Here we do not attempt to provide a complete discussion of this procedure, which for the (nonconformal) Sakai-Sugimoto model has been introduced only recently [71, 72]. We rather follow the method outlined in these papers and subtract a counterterm, fixed by a physical renormalization condition, as follows. After restricting the holographic integration in $\Omega_{b}$ to a finite interval $-\Lambda<z<\Lambda$, we subtract a counterterm $\delta \Omega_{b}(\Lambda)$ which cancels the divergence and obtain a renormalized contribution $\Omega_{b}^{\text {ren }}$. We also include a finite counterterm which is fixed by requiring the free energy in the absence of any chemical potential to vanish,

$$
\Omega\left(\mu_{B, I}=0\right)=0 .
$$

This condition is motivated by the observation that $\Omega$ should be the matter part of the free energy, i.e., it should describe the fermions and their interaction with the magnetic field. In particular, we thus require that the energy density of the (nondynamical) magnetic field in the absence of any matter be left out. This we shall later treat separately when we consider the Gibbs free energy (the Legendre transform from fixed internal magnetic field to fixed external magnetic field) in Sec. 4.4. The condition (3.15) implies that we have to require

$$
0=\Omega_{b}^{\text {ren }} \equiv \lim _{\Lambda \rightarrow \infty}\left[\Omega_{b}(\Lambda)-\delta \Omega_{b}(\Lambda)\right]
$$

To find the exact form of the counterterms we first note that, for constant $\hat{b}(z)=\hat{\mathcal{B}}$ and $b(z)=\mathcal{B}$,

$$
\Omega_{b}(\Lambda)=3 \kappa\left(\hat{\mathcal{B}}^{2}+\mathcal{B}^{2}\right)\left[\frac{\Lambda^{1 / 3}}{u_{\mathrm{KK}}^{1 / 3}}-\sqrt{\pi} \frac{\Gamma(5 / 6)}{\Gamma(1 / 3)}+\mathcal{O}\left(\frac{u_{\mathrm{KK}}^{5 / 3}}{\Lambda^{5 / 3}}\right)\right] .
$$

The counterterm $\delta \Omega_{b}(\Lambda)$ should depend only on fields and geometric data on the slice $z=\Lambda$, in particular it should only involve the induced metric $\gamma_{\mu \nu}$ on the slice and not 
the complete metric $g$. By including appropriate factors of the dilaton [71, 72] and an appropriate numerical factor to fulfill the condition $(3.16)$ we find

$$
\delta \Omega_{b}(\Lambda)=\frac{R}{2}\left[\left(\frac{e^{\Phi}}{g_{s}}\right)^{1 / 3}-\frac{\sqrt{\pi} \Gamma(5 / 6)}{\Gamma(1 / 3)}\left(\frac{u_{\mathrm{KK}}}{R}\right)^{1 / 2}\left(\frac{e^{\Phi}}{g_{s}}\right)^{-1 / 3}\right] \mathcal{C}(\Lambda),
$$

with

$$
\begin{aligned}
\mathcal{C}(\Lambda) & \equiv-\frac{T_{8} V_{4}\left(2 \pi \alpha^{\prime}\right)^{2}}{2} e^{-\Phi} \sqrt{\gamma} \gamma^{\mu \nu} \gamma^{\rho \sigma} \operatorname{Tr}\left[\mathcal{F}_{\nu \rho} \mathcal{F}_{\sigma \mu}\right] \\
& =\frac{6 \kappa}{u_{\mathrm{KK}}^{1 / 4} R^{3 / 4}}\left[\frac{\Lambda^{1 / 6}}{u_{\mathrm{KK}}^{1 / 6}}+\mathcal{O}\left(\frac{1}{\Lambda^{11 / 6}}\right)\right],
\end{aligned}
$$

where we have used Eq. (A.4) and where the indices $\mu, \nu, \rho, \sigma$ run over $0,1,2,3$. With this counterterm, the term proportional to $\Lambda^{1 / 3}\left(\Lambda^{0}\right)$ in Eq. (3.17) is cancelled by the first (second) term in Eq. (3.18)

In the case of a magnetic field which is not constant in the bulk, the second term in $\Omega_{b}$ as given by Eq. (3.14) is also divergent, but its divergence comes from the integration over the spatial directions perpendicular to the magnetic field, regardless of whether the holographic $z$-integration is finite or not. Therefore, we cannot treat this term by the usual holographic renormalization and we interpret this divergence, when present, as a Meissner effect: a phase where a homogeneous magnetic field $\mathcal{B}_{\mathrm{em}}$, which fixes the boundary values of $\hat{b}(z)$ and $b(z)$, is only possible for non-constant functions in $z$, is infinitely penalized such that only $\mathcal{B}_{\mathrm{em}}=0$ is allowed. As we shall see, this will be the case for the charged pion condensate, to be discussed further in Sec. 4.2.2. At this point we already observe that the role of the spatial directions transverse to the magnetic field is no coincidence. It points to the necessity of currents in these directions which produce a magnetic field equal in magnitude but with opposite direction compared to the external magnetic field. This leads to a vanishing total magnetic field in the system, which is nothing but the Meissner effect for superconductors.

\section{Chirally broken phases in a magnetic field}

In this section we solve the equations of motion for the chirally broken phase. We shall distinguish between two different chirally broken phases, the $\sigma$ and the $\pi$ phase. This is the main part of the paper, and the main physical results can be found in Secs. 4.3 and 4.4 .

\subsection{Chiral rotations and resulting boundary conditions}

In $N_{f}=2$ chiral perturbation theory the chiral field $U \in U(2)$ describing the Goldstone bosons is given by

$$
U=e^{i\left(\eta+\varphi_{a} \tau_{a}\right) / f_{\pi}},
$$

where $f_{\pi}$ is the pion decay constant (in the Sakai-Sugimoto model, $f_{\pi}=2 M_{\mathrm{KK}} \sqrt{\kappa / \pi}$ [17, 43]). The $\eta$ meson (the $\eta^{\prime}$ for $N_{f}=3$ ) becomes massive in QCD due to the explicit 
breaking of the $U(1)_{A}$ through the axial anomaly. This is realized in the Sakai-Sugimoto model through the Chern-Simons term, and the mass of the $\eta$ can be computed within the model, $m_{\eta}=\lambda M_{\mathrm{KK}} \sqrt{N_{f} / N_{c}} /(3 \sqrt{3} \pi)$ [17], see also Refs. [73, 74, 75, 76].

In the Sakai-Sugimoto model the chiral field is given by the holonomy 17]

$$
U=P \exp \left(i \int_{-\infty}^{\infty} d z \mathcal{A}_{z}\right)
$$

As mentioned above, we work in a gauge where $\mathcal{A}_{z}=0$. This is only possible by using the full $U(2)_{L} \times U(2)_{R}$ symmetry, implicitly taking into account a $\theta$ term, see also comment below Eq. (4.6). It seems we can then only consider the vacuum $U=\mathbf{1}$. However, we can keep the $\mathcal{A}_{z}=0$ gauge and recover other vacua encoded in the boundary values of the gauge fields. This is explained in detail for instance in Ref. [66]. We shall now recapitulate this explanation and apply it to our case.

Consider a potential $V\left[\mu_{L}, \mu_{R}, U(\phi)\right]$ which is invariant under $U\left(N_{f}\right)_{L} \times U\left(N_{f}\right)_{R}$. Here, $\mu_{L}, \mu_{R} \in U\left(N_{f}\right)$ are fixed external parameters. For the following argument we denote these parameters simply by $\mu_{L}, \mu_{R}$, reminiscent of the chemical potentials, but one should keep in mind that this notation also includes the magnetic field. The chiral field $U$ is written as a function of a parameter $\phi$ with respect to which we have to minimize the potential to find the vacuum. This parameter is a symbol for the meson fields in Eq. (4.1). The external parameters transform under the global symmetry as $\mu_{L} \rightarrow g_{L}^{-1} \mu_{L} g_{L}, \mu_{R} \rightarrow g_{R}^{-1} \mu_{R} g_{R}$, while the chiral field transforms as $U \rightarrow g_{L}^{-1} U g_{R}$, where $g_{L} \in U\left(N_{f}\right)_{L}, g_{R} \in U\left(N_{f}\right)_{R}$. Via a global symmetry transformation we have $V\left[\mu_{L}, \mu_{R}, U(\phi)\right]=V\left[g_{L}^{-1}(\phi) \mu_{L} g_{L}(\phi), g_{R}^{-1}(\phi) \mu_{R} g_{R}(\phi), \mathbf{1}\right]$ with $\phi$-dependent transformations $g_{L}(\phi), g_{R}(\phi)$ such that $g_{L}^{-1}(\phi) U(\phi) g_{R}(\phi)=1$. To find the vacuum of the theory it obviously does not matter whether we use the original potential or the potential with the transformed quantities because both expressions are simply identical. Consequently, instead of keeping the external parameters fixed and varying the chiral field we can fix the chiral field to be the unit matrix and vary the external parameters. Of course we cannot simply treat the external parameters as arbitrary continuous quantities with respect to which we minimize the potential. We need to ensure that they are connected by a transformation to their physical values. We shall see below that within our ansatz the allowed rotated parameters only assume two discrete values, such that we simply have to compare two separate phases with each other. After minimization of the potential, the physical vacuum is given by applying the rotation found from minimization "backwards" onto the unit matrix, i.e.,

$$
U=g_{L} g_{R}^{-1}
$$

Without loss of generality we can set $g_{R}=\mathbf{1}$ and thus $U=g_{L}$. We can write

$$
g_{L}=e^{i\left(\eta+\varphi_{a} \tau_{a}\right) / f_{\pi}}=e^{i \eta / f_{\pi}} \frac{\sigma+i \pi_{a} \tau_{a}}{f_{\pi}},
$$

where $\sigma / f_{\pi} \equiv \cos \left(\varphi / f_{\pi}\right), \pi_{a} / f_{\pi}=\varphi_{a} / \varphi \sin \left(\varphi / f_{\pi}\right)$ with $\varphi \equiv\left(\varphi_{1}^{2}+\varphi_{2}^{2}+\varphi_{3}^{2}\right)^{1 / 2}$. This is the usual form of the chiral field in chiral perturbation theory, where the massive mode, the "sigma", is frozen and the effective theory describes the remaining meson modes. Therefore, 


\begin{tabular}{|c||c|c|c|c|c|c|}
\hline & $\hat{A}_{0}( \pm \infty)$ & $A_{0}( \pm \infty)$ & $\hat{b}( \pm \infty)$ & $b( \pm \infty)$ & $\hat{A}_{3}( \pm \infty)$ & $A_{3}( \pm \infty)$ \\
\hline \hline$\sigma$ & $2 \mu_{B}$ & $2 \mu_{I}$ & $\hat{\mathcal{B}}$ & $\mathcal{B}$ & $\pm 2 \hat{\jmath}$ & $\pm 2 \jmath$ \\
\hline$\pi$ & $2 \mu_{B}$ & $\pm 2 \mu_{I}$ & $\hat{\mathcal{B}}(0)$ & $\pm \mathcal{B}(0)$ & $\pm 2 \hat{\jmath}$ & 0 \\
\hline
\end{tabular}

Table 1: Boundary conditions in the sigma and pion phases. The boundary conditions for the temporal components of the gauge fields correspond to the baryon and isospin chemical potentials, while the boundary conditions for the field strengths $\hat{F}_{12} \equiv \hat{b}, F_{12} \equiv b$ correspond to the baryon and isospin components of the magnetic field. The boundary conditions for the spatial components $\hat{A}_{3}$, $A_{3}$ are given by the meson supercurrents $\hat{\jmath}, \jmath$. These currents are not external parameters but have to be determined by minimizing the free energy. In the $\pi$ phase $A_{3}( \pm \infty)$ has to vanish to ensure a well-defined behavior of the gauge fields under parity transformations, see Eq. (C.6) and discussion above this equation. For a discussion of the normalization of the chemical potentials see Eq. (4.34) and below. The zeros in parantheses for the magnetic fields in the charged pion condensed phase indicate that eventually we shall set $\hat{\mathcal{B}}=\mathcal{B}=0$ because of the Meissner effect in this phase, see Sec. 4.2.2.

both sides of Eq. (4.4) contain four degrees of freedom; for the right-hand side we have the condition $\sigma^{2}+\pi^{2}=f_{\pi}^{2}$ which is obvious from the definitions of $\sigma$ and $\pi_{a}$.

To apply a rotation given by $g_{L}$ on the (left-handed) external parameter $\mu_{L}$ note that our physical chemical potentials and the magnetic field are diagonal in flavor space and identical for $L$ and $R, \mu_{L}=\mu_{R}=\mu_{B} \mathbf{1}+\mu_{I} \tau_{3}, \mathcal{B}_{\mathrm{em}, L}=\mathcal{B}_{\mathrm{em}, R}=\hat{\mathcal{B}} \mathbf{1}+\mathcal{B} \tau_{3}$. The baryon part $\propto \mathbf{1}$ does obviously not change under a $U(2)$ transformation. We thus only have to consider how the isospin part $\propto \tau_{3}$ transforms. We find

$$
g_{L}^{-1} \tau_{3} g_{L}=\frac{1}{f_{\pi}}\left[\pi^{+}\left(\pi^{0}+i \sigma\right) \tau_{+}+\pi^{-}\left(\pi^{0}-i \sigma\right) \tau_{-}+\left(1-2 \pi^{+} \pi^{-}\right) \tau_{3}\right],
$$

where $\tau_{ \pm} \equiv \tau_{1} \pm i \tau_{2}$, and where we have introduced the neutral pion $\pi^{0}=\pi_{3}$ and the charged pions $\pi^{ \pm} \equiv \pi_{1} \mp i \pi_{2}$. In our ansatz described in Sec. 3.1 we have restricted ourselves to diagonal gauge fields. Since the chemical potentials and the magnetic field are the boundary values for the gauge fields, they have to be diagonal too. Consequently, we can only allow for transformations (4.5) that transform $\tau_{3}$ into a matrix $\propto \tau_{3}$. There are two (nontrivial) possibilities to make the coefficients in front of $\tau_{+}, \tau_{-}$vanish: $(i) \pi^{+}=\pi^{-}=0$ which leads to $g_{L}^{-1} \tau_{3} g_{L}=\tau_{3}$ and $(i i) \pi^{0}=\sigma=0$ which leads to $g_{L}^{-1} \tau_{3} g_{L}=-\tau_{3}$. Hence we can either leave the isospin components of the chemical potentials and the magnetic field invariant or flip their sign. This means that the parameter $\phi$ in $U(\phi)$ above is in fact discrete, not continuous. Had we allowed for off-diagonal components in the gauge fields, we could have described arbitrary linear combinations of the pion fields.

These somewhat formal arguments have a very intuitive geometric interpretation 65]: another (simpler, but less precise) way of saying what we have just explained is the following. Think of the D8 branes as a left-handed up-brane and a left-handed down-brane and of the $\overline{\mathrm{D} 8}$ branes as a right-handed up-brane and a right-handed down-brane. Then, a chirally broken phase can be constructed by connecting $(i)$ the left-handed up-brane with the right-handed up-brane and likewise for the down-branes or $(i i)$ the left-handed up-brane with the right-handed down-brane and vice versa. These two possibilities corre- 
spond exactly to the two cases from the above formal argument: case $(i)$ corresponds to a condensate where equal quark flavors participate, i.e., a combination of $\sigma$ and $\pi^{0}$ with $\bar{u}-u$ and $\bar{d}-d$ pairing. In the remainder of the paper we shall refer to this case as the $\sigma$ phase. Case (ii) corresponds to a charged pion condensate with nonzero $\left\langle\bar{d} \gamma_{5} u\right\rangle,\left\langle\bar{u} \gamma_{5} d\right\rangle$, to which we shall refer as the $\pi$ phase (this phase is sometimes called " $\rho$ " 65, 77]). As a summary of this section and a reminder for the subsequent sections, we present the resulting boundary conditions for the $\sigma$ and the $\pi$ phases in Table 1 .

In this Table we have also included the supercurrents $\hat{\jmath}$, , which, in our gauge $g_{L}=U$, have the form $g_{L}^{-1} \nabla g_{L}$ [17]. With Eq. (4.4) this becomes for the two phases

$$
\begin{array}{ll}
\text { (i) } \sigma \text { phase : } & -i g_{L}^{-1} \nabla g_{L}=\frac{\nabla \eta}{f_{\pi}}+\frac{\tau_{3}}{f_{\pi}^{2}}\left(\sigma \nabla \pi^{0}-\pi^{0} \nabla \sigma\right), \\
\text { (ii) } \pi \text { phase : } & -i g_{L}^{-1} \nabla g_{L}=\frac{\nabla \eta}{f_{\pi}}+\frac{i \tau_{3}}{2 f_{\pi}^{2}}\left(\pi^{-} \nabla \pi^{+}-\pi^{+} \nabla \pi^{-}\right) .
\end{array}
$$

We see that the supercurrents are diagonal, i.e., our ansatz with nonvanishing 1-component $\hat{\jmath}$ and $\tau_{3}$-component $\jmath$ is consistent. Interestingly, an anisotropic $\eta$ condensate appears in the 1-components $\hat{\jmath}$. The $\eta$ condensate has dropped out in Eq. (4.5), and thus our boundary conditions, given by the chemical potentials and the magnetic field modified by the rotation (4.5), do not reveal whether there is an admixture of an $\eta$ condensate in the $\sigma$ phase. On the other hand, a nonzero supercurrent $\hat{\jmath}$ seems to indicate the presence of an $\eta$ supercurrent. Indeed, we shall see later that in the $\sigma$ phase a nonzero $\hat{\jmath}$ is induced. The term $\nabla \eta$ in Eqs. (4.6) appears due to our use of the full $U(2)_{L} \times U(2)_{R}$ symmetry. Strictly speaking, our Lagrangian breaks the axial $U(1)_{A}$ because of the presence of the Chern-Simons term. However, this symmetry is preserved if one compensates a $U(1)_{A}$ rotation by a shift of the $\theta$ parameter, whose realization in the Sakai-Sugimoto model is discussed in Ref. [17, 75, 76], see also Ref. [78]. We thus implicitly adjust the $\theta$ parameter when using the full gauge symmetry, absorbing a constant $\eta$ mode into $\theta$. We shall proceed within this simplification, but have to keep in mind that in a more complete approach one would have to consider a fixed $\theta$ and allow for a constant $\eta$ mode explicitly. Such an approach would be of interest especially in view of recent studies of possible (CP-violating) $\eta$ condensates in an NJL model calculation [79], or, including a magnetic field, in the linear sigma model [12].

We finally remark that our setup does not include the possibility of diquark condensation of the form $\langle u d\rangle$, which is expected to lead to color superconductivity of quark matter at sufficiently large baryon chemical potential [80]. However, color superconductivity does not necessarily occur in the large $N_{c}$ limit where a "chiral density wave" is a strong candidate for the ground state [81, 82], or, as suggested recently, quark matter may be confined even for large chemical potentials [59, 83].

\subsection{Solutions of the equations of motion and free energies}

We can now solve the equations of motion (3.4) and (3.6) for the two sets of boundary conditions given in Table 11. For notational convenience we set $u_{\mathrm{KK}}=1$ (the final results in Secs. 4.3 and 4.4 do not depend on $u_{\mathrm{KK}}$ and thus all physical quantities will have the 
correct dimensions). For both sets of boundary conditions we first note that the differential equations (3.6) can be solved by defining the new functions

$$
F_{0}^{ \pm}(z) \equiv k(z) \frac{\hat{F}_{z 0} \pm F_{z 0}}{2}, \quad F_{3}^{ \pm}(z) \equiv k(z) \frac{\hat{F}_{z 3} \pm F_{z 3}}{2}
$$

Then, the four equations (3.6) are equivalent to

$$
\partial_{z} F_{0}^{ \pm}=\frac{\alpha[\hat{b}(z) \pm b(z)]}{k(z) M_{\mathrm{KK}}^{2}} F_{3}^{ \pm}(z), \quad \partial_{z} F_{3}^{ \pm}=\frac{\alpha[\hat{b}(z) \pm b(z)]}{k(z) M_{\mathrm{KK}}^{2}} F_{0}^{ \pm}(z) .
$$

Now the two equations with the upper sign are decoupled from the two equations with the lower sign. To proceed, we have to distinguish between the two chirally broken phases.

\subsubsection{Sigma phase}

With the boundary conditions of the $\sigma$ phase from Table 11 and with Eqs. (3.4) we conclude that the magnetic fields are constant in the bulk

$$
\hat{b}(z)=\hat{\mathcal{B}}, \quad b(z)=\mathcal{B} .
$$

In the following, we shall denote the dimensionless magnetic fields by

$$
\hat{B} \equiv \frac{\alpha \hat{\mathcal{B}}}{M_{\mathrm{KK}}^{2}}, \quad B \equiv \frac{\alpha \mathcal{B}}{M_{\mathrm{KK}}^{2}}, \quad B_{\mathrm{em}} \equiv \frac{\alpha \mathcal{B}_{\mathrm{em}}}{M_{\mathrm{KK}}^{2}} .
$$

We can now solve Eqs. (4.8) for completely general boundary conditions for the gauge fields. This is done in Appendix B, where we present some technical details. Here we proceed with the specific solution obtained from the boundary conditions given in the first row of Table 1. This solution yields the gauge fields

$$
\begin{aligned}
& \hat{A}_{0}(z)=2 \mu_{B}+\hat{\jmath}\left[C_{+}(z)+C_{-}(z)-T_{+}\right]+\jmath\left[C_{+}(z)-C_{-}(z)-T_{-}\right], \\
& A_{0}(z)=2 \mu_{I}+\jmath\left[C_{+}(z)+C_{-}(z)-T_{+}\right]+\hat{\jmath}\left[C_{+}(z)-C_{-}(z)-T_{-}\right], \\
& \hat{A}_{3}(z)=\hat{\jmath}\left[S_{+}(z)+S_{-}(z)\right]+\jmath\left[S_{+}(z)-S_{-}(z)\right], \\
& A_{3}(z)=\jmath\left[S_{+}(z)+S_{-}(z)\right]+\hat{\jmath}\left[S_{+}(z)-S_{-}(z)\right],
\end{aligned}
$$

where we have abbreviated

$$
\begin{aligned}
C_{ \pm}(z) & \equiv \frac{\cosh [(\hat{B} \pm B) \arctan z]}{\sinh [\pi(\hat{B} \pm B) / 2]}, \quad S_{ \pm}(z) \equiv \frac{\sinh [(\hat{B} \pm B) \arctan z]}{\sinh [\pi(\hat{B} \pm B) / 2]} \\
T_{ \pm} & \equiv \operatorname{coth} \frac{\pi(\hat{B}+B)}{2} \pm \operatorname{coth} \frac{\pi(\hat{B}-B)}{2}
\end{aligned}
$$

Note that $T_{ \pm}=C_{+}(\infty) \pm C_{-}(\infty)=C_{+}(-\infty) \pm C_{-}(-\infty)$. Since the functions $C_{ \pm}(z)$ and $S_{ \pm}(z)$ are symmetric and antisymmetric in $z$, respectively, both temporal components of the gauge fields are symmetric while both spatial components are antisymmetric. Together

with the behavior of the supercurrents under a parity transformation this ensures that the gauge fields transform as a vector under parity, see discussion below Eq. (B.9). We plot 

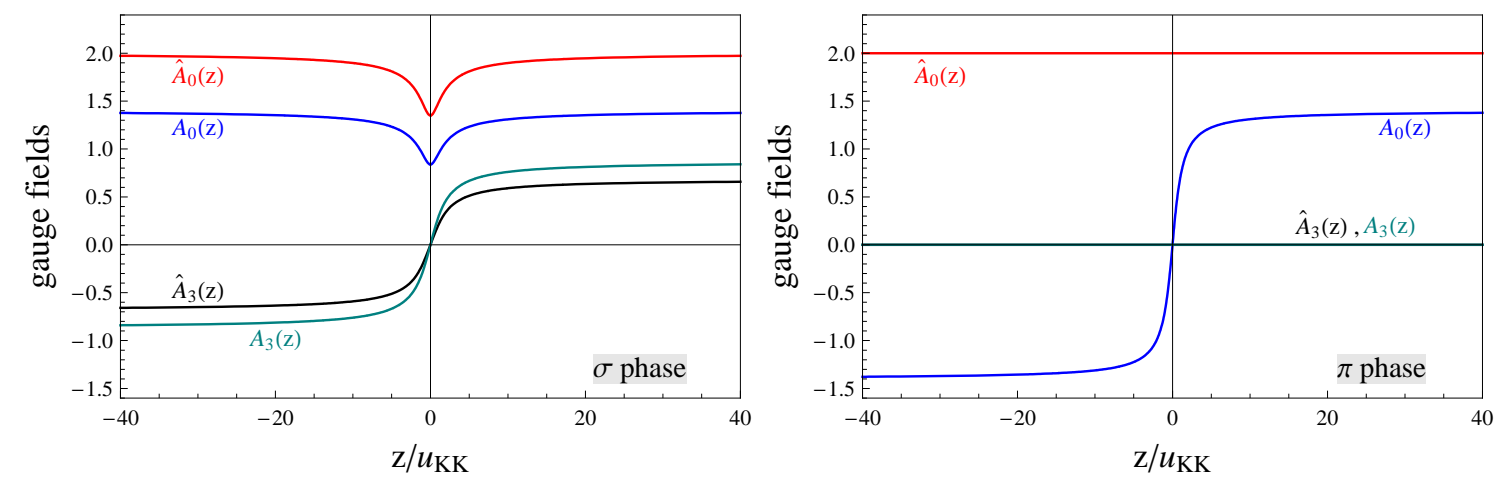

Figure 1: Energetically preferred configuration of the gauge fields as a function of the holographic coordinate $z$ for the sigma phase (left panel) and the charged pion phase (right panel). For the sigma phase we have chosen a dimensionless magnetic field $e B_{\mathrm{em}}=2$. In the pion phase, $B_{\mathrm{em}}=0$ due to the Meissner effect. The boundary values for $\hat{A}_{0}(z)$ and $A_{0}(z)$ are given by (twice) the baryon and isospin chemical potentials, respectively. The boundary values of $\hat{A}_{3}(z)$ and $A_{3}(z)$ yield the meson supercurrents and are determined dynamically from minimization of the free energy.

the gauge fields with the supercurrents determined from minimization of the free energy, see Eqs. 4.15), in the left panel of Fig. 11.

Next, we insert the gauge fields and the resulting field strengths into the free energy (3.13). We drop the contributions $\Omega_{g}$ and, via holographic renormalization, $\Omega_{b}$, as explained in Sec. 3.2. Then we obtain (for details see Appendix B)

$$
\begin{aligned}
\Omega=\frac{2 \kappa M_{\mathrm{KK}}^{2}}{3} & {\left[(\hat{\jmath}+\jmath)^{2} \rho_{+}(\hat{B}, B)+(\hat{\jmath}-\jmath)^{2} \rho_{-}(\hat{B}, B)\right.} \\
- & \left.4 \mu_{B}(\hat{\jmath} \hat{B}+\jmath B)-4 \mu_{I}(\hat{\jmath} B+\jmath \hat{B})\right]
\end{aligned}
$$

with

$$
\rho_{ \pm}(\hat{B}, B) \equiv 2(\hat{B} \pm B) \operatorname{coth} \frac{\pi(\hat{B} \pm B)}{2}+\frac{\pi(\hat{B} \pm B)^{2}}{2 \sinh ^{2}[\pi(\hat{B} \pm B) / 2]} .
$$

The asymptotic values of the functions $\rho_{ \pm}(\hat{B}, B)$ at small and large magnetic fields are shown in Table 2 in Appendix B.

Minimizing $\Omega$ with respect to $\hat{\jmath}$, yields

$$
\begin{aligned}
& \hat{\jmath}=\frac{\mu_{B}+\mu_{I}}{2} \frac{\hat{B}+B}{\rho_{+}(\hat{B}, B)}+\frac{\mu_{B}-\mu_{I}}{2} \frac{\hat{B}-B}{\rho_{-}(\hat{B}, B)}, \\
& \jmath=\frac{\mu_{B}+\mu_{I}}{2} \frac{\hat{B}+B}{\rho_{+}(\hat{B}, B)}-\frac{\mu_{B}-\mu_{I}}{2} \frac{\hat{B}-B}{\rho_{-}(\hat{B}, B)} .
\end{aligned}
$$

One can check that this is indeed a minimum of $\Omega$ : the matrix of second derivatives of $\Omega$ with respect to the supercurrents has eigenvalues $8 \kappa M_{\mathrm{KK}}^{2} \rho_{+} / 3,8 \kappa M_{\mathrm{KK}}^{2} \rho_{-} / 3$, which are independent of $\hat{\jmath}$ and $\jmath$ and positive for all $\hat{B}, B$. 
As already mentioned below Eq. (3.3) we recall that the supercurrents $\hat{\jmath}, \jmath$ act as a source for the normal currents $\hat{\mathcal{J}}, \mathcal{J}$ which are the spatial 3-components of the four-currents

$$
\mathcal{J}_{R / L}^{\mu}=\mathcal{J}_{\mathrm{YM}, R / L}^{\mu}+\mathcal{J}_{\mathrm{CS}, R / L}^{\mu}
$$

where the Yang-Mills and Chern-Simons contributions are given by 43, 69]

$$
\begin{aligned}
\mathcal{J}_{\mathrm{YM}, R / L}^{\mu} & =\left.\mp 2 \kappa M_{\mathrm{KK}}^{2} k(z) \mathcal{F}^{\mu z}\right|_{z= \pm \infty}, \\
\mathcal{J}_{\mathrm{CS}, R / L}^{\mu} & =\left.\mp \frac{N_{c}}{24 \pi^{2}} \epsilon^{\mu \nu \rho \sigma} \mathcal{A}_{\nu} \mathcal{F}_{\rho \sigma}\right|_{z= \pm \infty} .
\end{aligned}
$$

Here, the indices $\mu, \nu, \rho, \sigma$ run over $0,1,2,3$, the upper (lower) signs correspond to $R(L)$, and we have, in the Chern-Simons contribution, already used that in our ansatz the offdiagonal components of the gauge fields in flavor space vanish. With the gauge fields (4.11) and the field strengths (B.9) we obtain the baryon and isospin components of the spatial currents,

$$
\begin{aligned}
& \hat{\mathcal{J}} \equiv \hat{\mathcal{J}}_{R}=-\hat{\mathcal{J}}_{L}=\kappa M_{\mathrm{KK}}^{2}\left[\left(\mu_{B}+\mu_{I}\right) \frac{(\hat{B}+B)^{2}}{\rho_{+}(\hat{B}, B)} \operatorname{coth} \frac{\pi(\hat{B}+B)}{2}\right. \\
&\left.+\left(\mu_{B}-\mu_{I}\right) \frac{(\hat{B}-B)^{2}}{\rho_{-}(\hat{B}, B)} \operatorname{coth} \frac{\pi(\hat{B}-B)}{2}-\frac{2}{3}\left(\mu_{B} \hat{B}+\mu_{I} B\right)\right], \\
& \mathcal{J} \equiv \mathcal{J}_{R}=-\mathcal{J}_{L}=\kappa M_{\mathrm{KK}}^{2}\left[\left(\mu_{B}+\mu_{I}\right) \frac{(\hat{B}+B)^{2}}{\rho_{+}(\hat{B}, B)} \operatorname{coth} \frac{\pi(\hat{B}+B)}{2}\right. \\
&\left.-\left(\mu_{B}-\mu_{I}\right) \frac{(\hat{B}-B)^{2}}{\rho_{-}(\hat{B}, B)} \operatorname{coth} \frac{\pi(\hat{B}-B)}{2}-\frac{2}{3}\left(\mu_{B} B+\mu_{I} \hat{B}\right)\right],
\end{aligned}
$$

where the terms with prefactor $2 / 3$ are the Chern-Simons contributions. These currents are already evaluated at the minimum of the free energy, i.e., we have inserted the supercurrents (4.15). They add up to zero in the sums $\mathcal{J}_{L}+\mathcal{J}_{R}$ and $\hat{\mathcal{J}}_{L}+\hat{\mathcal{J}}_{R}$, corresponding to vanishing baryon and isospin currents, however they yield nonzero axial currents.

For small magnetic fields, the linear terms of the Yang-Mills and Chern-Simons contributions cancel exactly and thus the currents become cubic in the magnetic field,

$$
\begin{aligned}
& \hat{\mathcal{J}} \simeq \frac{\kappa M_{\mathrm{KK}}^{2} \pi^{2}}{54}\left[(\hat{B}+B)^{3}\left(\mu_{B}+\mu_{I}\right)+(\hat{B}-B)^{3}\left(\mu_{B}-\mu_{I}\right)\right], \\
& \mathcal{J} \simeq \frac{\kappa M_{\mathrm{KK}}^{2} \pi^{2}}{54}\left[(\hat{B}+B)^{3}\left(\mu_{B}+\mu_{I}\right)-(\hat{B}-B)^{3}\left(\mu_{B}-\mu_{I}\right)\right] .
\end{aligned}
$$

The cancellation of the linear terms seems to suggest that there might also be cancellations in the cubic terms from terms we have neglected upon expanding the DBI action for small gauge fields, possibly leading to vanishing currents. By considering a one-flavor system (where the use of the full DBI action is much simpler) we have checked that this is not the case. $^{3}$

\footnotetext{
${ }^{3}$ This is at variance with Ref. [49] where a vanishing axial current has been found. In this reference, however, a modified action has been used where certain surface terms are dropped, essentially to force the current to vanish. Since we do not see any contradiction in a nonvanishing current (in the presence of an external magnetic field) we leave the full resolution of this discrepancy to future studies.
} 
We plot the currents $\hat{\mathcal{J}}, \mathcal{J}$ in Fig. 2, where we also show the supercurrents and the densities in the sigma phase. We remark that the expansion of the 3-component of the gauge fields does not contain the complete current. One rather only finds the Yang-Mills contribution as a coefficient in front of the next-to-leading term of the asymptotic expansion,

$$
\hat{A}_{3}(z)= \pm 2 \hat{\jmath} \mp \frac{\hat{\mathcal{J}}_{\mathrm{YM}, R / L}}{\kappa M_{\mathrm{KK}}^{2}} \frac{1}{z}+\mathcal{O}\left(\frac{1}{z^{2}}\right), \quad A_{3}(z)= \pm 2 \jmath \mp \frac{\mathcal{J}_{\mathrm{YM}, R / L}}{\kappa M_{\mathrm{KK}}^{2}} \frac{1}{z}+\mathcal{O}\left(\frac{1}{z^{2}}\right) .
$$

Finally, inserting the values (4.15) back into $\Omega$ yields the value of the free energy at the minimum,

$$
\Omega_{\sigma}=-\frac{2 \kappa M_{\mathrm{KK}}^{2}}{3}\left[\left(\mu_{B}+\mu_{I}\right)^{2} \frac{(\hat{B}+B)^{2}}{\rho_{+}(\hat{B}, B)}+\left(\mu_{B}-\mu_{I}\right)^{2} \frac{(\hat{B}-B)^{2}}{\rho_{-}(\hat{B}, B)}\right] .
$$

We see that for vanishing magnetic fields $\Omega_{\sigma}=0$, i.e., the free energy does not depend on any of the chemical potentials. This is the expected result for the sigma phase and has also been observed in Ref. [65].

\subsubsection{Pion phase and Meissner effect}

In this case the boundary conditions are given in the second row of Table 1, and the differential equations (3.4) for the magnetic fields have the solution

$$
\hat{b}(z)=\hat{\mathcal{B}}, \quad b(z)=\frac{2 \mathcal{B}}{\pi} \arctan z .
$$

As we have discussed at the end of Sec. 3.2, nonconstant functions $\hat{b}$ or $b$ lead to an infinite contribution to the free energy which cannot be removed by holographic renormalization, but which enforces a vanishing magnetic field, indicating a Meissner effect. In the $\pi$ phase, it is the nonconstant isospin component $b(z)$ which leads to this conclusion. This is only to be expected since the condensate of pions carries an electric charge, and thus the system is an electromagnetic superconductor. By the Meissner effect, a magnetic field is induced which is opposite, but equal in magnitude, to the applied magnetic field, such that $\mathcal{B}_{\mathrm{em}}=0$ and thus $\hat{B}=B=0$. Of course our electromagnetic group is only global and thus the microscopic description of the Meissner effect, for instance in terms of a Meissner mass for the photon, is not straightforward. However, in terms of supercurrents, the effect can be described quite naturally: in fact we have to allow for a supercurrent in the directions transverse to the magnetic field, i.e., $\boldsymbol{\jmath}_{s}(\mathbf{x}, z)=\frac{1}{2} b(z)\left(x_{2},-x_{1}, 0\right)$, such that curl $\boldsymbol{\jmath}_{s}=-b$ (and the same for the components $\left.\hat{\boldsymbol{j}}_{s}, \hat{b}\right)$. This is the usual London equation for a superconductor, see for instance Ref. 84]. Consequently, we need to add the supercurrents $\hat{\boldsymbol{j}}_{s}, \boldsymbol{\jmath}_{s}$ to the boundary conditions of the gauge fields $\hat{A}_{1}(\mathbf{x}, z), \hat{A}_{2}(\mathbf{x}, z), A_{1}(\mathbf{x}, z), A_{2}(\mathbf{x}, z)$ from Eqs. (3.3) such that the total boundary conditions (and thereby the total magnetic field in the superconductor) vanish, $\hat{B}=B=0$. This condition renders the equations of motion for the pion phase very simple. We shall, however, solve these equations for arbitrary magnetic

fields and only at the end set $\hat{B}=B=0$. This provides us with a better understanding of the structure of the solution, for instance its behavior under parity transformations. 
We defer all technical details and the solution for general boundary conditions with the magnetic fields (4.22) to Appendix $\mathrm{Q}$. For the specific boundary conditions characterizing the charged pion condensate we find the solutions

$$
\begin{aligned}
& \hat{A}_{0}(z)=2 \mu_{B}+\mu_{I}\left[\tilde{C}_{+}(z)+\tilde{C}_{-}(z)-\tilde{T}_{+}\right]+\hat{\jmath}\left[\tilde{C}_{+}(z)-\tilde{C}_{-}(z)-\tilde{T}_{-}\right], \\
& A_{0}(z)=\mu_{I}\left[\tilde{S}_{+}(z)+\tilde{S}_{-}(z)\right]+\hat{\jmath}\left[\tilde{S}_{+}(z)-\tilde{S}_{-}(z)\right], \\
& \hat{A}_{3}(z)=\hat{\jmath}\left[\tilde{S}_{+}(z)+\tilde{S}_{-}(z)\right]+\mu_{I}\left[\tilde{S}_{+}(z)-\tilde{S}_{-}(z)\right], \\
& A_{3}(z)=\hat{\jmath}\left[\tilde{C}_{+}(z)+\tilde{C}_{-}(z)-\tilde{T}_{-}\right]+\mu_{I}\left[\tilde{C}_{+}(z)-\tilde{C}_{-}(z)-\tilde{T}_{+}\right],
\end{aligned}
$$

where we abbreviated

$$
\begin{aligned}
\tilde{C}_{+}(z) & \equiv \frac{P_{+}(z)+P_{-}(z)}{P_{+}^{+}-P_{+}^{-}}, \quad \tilde{C}_{-}(z) \equiv \frac{Q_{+}(z)+Q_{-}(z)}{Q_{+}^{+}-Q_{+}^{-}}, \\
\tilde{S}_{+}(z) & \equiv \frac{P_{+}(z)-P_{-}(z)}{P_{+}^{+}-P_{+}^{-}}, \quad \tilde{S}_{-}(z) \equiv \frac{Q_{+}(z)-Q_{-}(z)}{Q_{+}^{+}-Q_{+}^{-}}, \\
\tilde{T}_{ \pm} & \equiv \frac{P_{+}^{+}+P_{+}^{-}}{P_{+}^{+}-P_{+}^{-}} \pm \frac{Q_{+}^{+}+Q_{+}^{-}}{Q_{+}^{+}-Q_{+}^{-}}
\end{aligned}
$$

with

$$
\begin{aligned}
& Q_{ \pm}(z) \equiv \frac{\pi}{2 \sqrt{B}} e^{\frac{\pi \hat{B}^{2}}{4 B}} \operatorname{erf}\left(\frac{\pi \hat{B} \pm 2 B \arctan z}{2 \sqrt{\pi B}}\right) \\
& P_{ \pm}(z) \equiv \frac{\pi}{2 \sqrt{B}} e^{-\frac{\pi \hat{B}^{2}}{4 B}} \operatorname{erfi}\left(\frac{\pi \hat{B} \pm 2 B \arctan z}{2 \sqrt{\pi B}}\right)
\end{aligned}
$$

and $Q_{+}^{ \pm} \equiv Q_{+}( \pm \infty), P_{+}^{ \pm} \equiv P_{+}( \pm \infty)$. Here, erf is the error function and erfi $(z) \equiv \operatorname{erf}(i z) / i$. The functions $\tilde{C}_{ \pm}, \tilde{S}_{ \pm}, \tilde{T}_{ \pm}$are the more complicated counterparts of the functions $C_{ \pm}, S_{ \pm}$, $T_{ \pm}$from Eqs. (4.12). They share the same property $\tilde{T}_{ \pm}=\tilde{C}_{+}(\infty) \pm \tilde{C}_{-}(\infty)=\tilde{C}_{+}(-\infty) \pm$ $\tilde{C}_{-}(-\infty)$, and, as their counterparts, $\tilde{C}_{ \pm}(z)$ and $\tilde{S}_{ \pm}(z)$ are symmetric and antisymmetric in $z$, respectively. This means that the temporal 1-component and the spatial $\tau_{3}$-component are symmetric in $z$ while the temporal $\tau_{3}$-component and the spatial-1 component are antisymmetric. Again, together with the parity transformations of the supercurrents, this gives the correct parity behavior of the gauge fields, see discussion in Appendix Q. In particular, the requirement of a well-defined parity leads to the condition $\jmath=0$.

Inserting Eqs. (4.23) and the corresponding field strengths into the free energy (3.13) yields

$$
\Omega=\frac{2 \kappa M_{\mathrm{KK}}^{2}}{3}\left\{\left(\hat{\jmath}^{2}-\mu_{I}^{2}\right) \rho(\hat{B}, B)-2 \mu_{B}\left[\mu_{I} \eta_{+}(\hat{B}, B)+\hat{\jmath} \eta_{-}(\hat{B}, B)\right]\right\}+\Omega_{b},
$$

with $\Omega_{b}$ given in Eq. (3.14) and

$$
\begin{aligned}
\rho(\hat{B}, B) & \equiv \frac{4 \pi}{\left(P_{+}^{+}-P_{+}^{-}\right)\left(Q_{+}^{+}-Q_{+}^{-}\right)}+4 \cosh \frac{\pi \hat{B}}{2}\left(\frac{e^{\frac{\pi B}{4}}}{P_{+}^{+}-P_{+}^{-}}+\frac{e^{-\frac{\pi B}{4}}}{Q_{+}^{+}-Q_{+}^{-}}\right) \\
\eta_{ \pm}(\hat{B}, B) & \equiv 2 \sinh \frac{\pi \hat{B}}{2}\left(\frac{e^{\frac{\pi B}{4}}}{P_{+}^{+}-P_{+}^{-}} \mp \frac{e^{-\frac{\pi B}{4}}}{Q_{+}^{+}-Q_{+}^{-}}\right)
\end{aligned}
$$


Again, the asymptotic values of these functions are given in Table 2 in Appendix B.

Minimization of $\Omega$ with respect to $\hat{\jmath}$ yields

$$
\hat{\jmath}=\mu_{B} \frac{\eta_{-}(\hat{B}, B)}{\rho(\hat{B}, B)},
$$

and the minimum of the free energy becomes

$$
\Omega_{\pi}=-\frac{2 \kappa M_{\mathrm{KK}}^{2}}{3}\left[\mu_{B}^{2} \frac{\eta_{-}^{2}(\hat{B}, B)}{\rho(\hat{B}, B)}+\mu_{I}^{2} \rho(\hat{B}, B)+2 \mu_{B} \mu_{I} \eta_{+}(\hat{B}, B)\right]+\Omega_{b} .
$$

We see that for vanishing magnetic fields the free energy depends on the isospin chemical potential, giving rise to a nonzero isospin density. This is expected from the quark content of the charged pion condensate and was also observed within the Sakai-Sugimoto model in Ref. 65].

Taking into account the Meissner effect, which is enforced by the infrared divergence in $\Omega_{b}$ as discussed at the beginning of this subsection, we have to set $\hat{B}=B=0$, leading to the simple result for the free energy

$$
\Omega_{\pi}=-\frac{8 \kappa M_{\mathrm{KK}}^{2}}{\pi} \mu_{I}^{2}
$$

From Eq. (4.28) we conclude that for $\hat{B}=B=0$ we have $\hat{\jmath}=0$. And, from the definitions (4.17), we see that in the absence of a magnetic field also the normal currents vanish, $\hat{\mathcal{J}}=\mathcal{J}=0$. In the following we shall discuss the results of the pion phase only in the presence of the Meissner effect.

\subsection{Meson supercurrents and number densities}

We have seen that all currents in the charged pion phase vanish (except for the supercurrents in the transverse 1- and 2-directions which cancel the applied magnetic field). The supercurrents and normal currents in the sigma phase, given in Eqs. (4.15) and (4.18), respectively, are shown in the left panel of Fig. 2 as a function of the magnetic field. We have used the electromagnetic field $B_{\mathrm{em}}$, defined in Eq. (3.2), with the electric charges of up and down quarks. We see that the supercurrents behave linear in $B_{\mathrm{em}}$ for small $B_{\mathrm{em}}$ and approach an asymptotic value for a large magnetic field. These limit cases assume very simple forms in terms of the electric quark charges $q_{i}$ and the quark chemical potentials $\mu_{1,2} \equiv \mu_{B} \pm \mu_{I}$. The quark supercurrents $\jmath_{1,2} \equiv \hat{\jmath} \pm \jmath$ in the sigma phase then are

$$
J_{i}^{\sigma} \simeq \frac{1}{2}\left\{\begin{array}{cc}
\frac{\pi q_{i} \mu_{i} B_{\mathrm{em}}}{3} & \text { for small } B_{\mathrm{em}} \\
\mu_{i} \operatorname{sgn} q_{i} & \text { for large } B_{\mathrm{em}}
\end{array} .\right.
$$

The limit of a large magnetic field is strictly speaking not consistent with our approximations. Firstly, we have expanded the DBI action for small gauge fields. Secondly, we have treated the flavor branes as probe branes which becomes questionable for large magnetic fields since one would have to consider the backreaction on the background geometry. As 

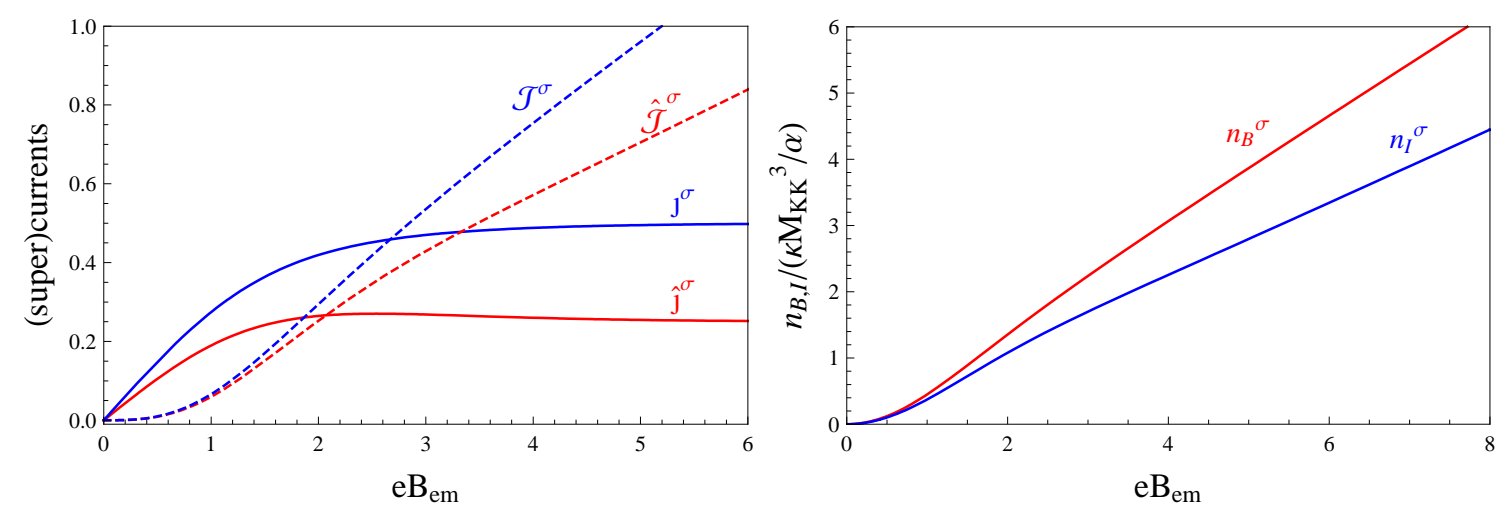

Figure 2: Left panel: Meson supercurrents $\hat{\jmath}^{\sigma}$ (red solid) and $\jmath^{\sigma}$ (blue solid) and normal currents $\hat{\mathcal{J}}^{\sigma}$ (red dashed) and $\mathcal{J}^{\sigma}$ (blue dashed) as a function of the dimensionless magnetic field $e B_{\mathrm{em}}$ in the sigma phase. The units are $M_{\mathrm{KK}} / \alpha$ for $\hat{\jmath}^{\sigma}, \jmath^{\sigma}$ and $\kappa M_{\mathrm{KK}}^{3} / \alpha$ for $\hat{\mathcal{J}}^{\sigma}, \mathcal{J}^{\sigma}$. Right panel: baryon and isospin number densities as a function of the dimensionless magnetic field in the sigma phase. The analytical expressions for the functions are given in Eqs. (4.15), (4.18), and (4.33). We have fixed $\mu_{B}=2 \mu_{I}=M_{\mathrm{KK}} \alpha$. In the (charged) pion phase, all currents as well as the baryon density vanish due to the Meissner effect; the isospin density is given by the simple expression (4.36b).

mentioned in Ref. [50] in the same context, the case of a large magnetic field within the present approach can only be meaningful if one thinks of the action (2.11) as a "bottomup" model for QCD, which is not derived from an AdS/CFT correspondence. Indeed, with appropriate functions $k(z), h(z)$, the bottom-up model from Ref. [26] can be recovered from Eq. (3.8). Thus, in the following we shall use our analytical functions to discuss the whole range of magnetic fields with this qualification in mind.

We can compute the baryon and isospin densities from the free energies computed in the previous sections via

$$
n_{B, I}=-\frac{\partial \Omega}{\partial \mu_{B, I}}
$$

We obtain for the sigma phase

$$
\begin{aligned}
& n_{B}^{\sigma}=\frac{4 \kappa M_{\mathrm{KK}}^{2}}{3}\left[\left(\mu_{B}+\mu_{I}\right) \frac{(\hat{B}+B)^{2}}{\rho_{+}}+\left(\mu_{B}-\mu_{I}\right) \frac{(\hat{B}-B)^{2}}{\rho_{-}}\right], \\
& n_{I}^{\sigma}=\frac{4 \kappa M_{\mathrm{KK}}^{2}}{3}\left[\left(\mu_{B}+\mu_{I}\right) \frac{(\hat{B}+B)^{2}}{\rho_{+}}-\left(\mu_{B}-\mu_{I}\right) \frac{(\hat{B}-B)^{2}}{\rho_{-}}\right],
\end{aligned}
$$

which agrees with the 0-component of the four-current defined in Eq. (4.16),

$$
\mathcal{J}_{R}^{0}+\mathcal{J}_{L}^{0}=-\frac{\partial \Omega}{\partial \mu_{B}}-\frac{\partial \Omega}{\partial \mu_{I}} \tau_{3}
$$

provided we use $2 \mu_{B, I}$ (as opposed to $\mu_{B, I}$ ) for the boundary values of $\hat{A}_{0}, A_{0}$, see Table 1 . Remarkably, with these simple rescalings we can reconcile the thermodynamic interpretation of $\Omega$ as a free energy with the standard definition of the currents from gauge/gravity 
duality. The reason for this unconventional normalization appears to be the anomalous nature of baryon and isospin number in the present model ${ }^{4}$.

As for the 3-components of the gauge fields (4.20), also the next-to-leading terms in the asymptotic expansions of the 0-components only contain the Yang-Mills contribution $\mathcal{J}_{\text {YM }}^{0}$ of Eq. (4.17a).

The densities are plotted in the right panel of Fig. 2. As expected, both densities vanish in the case of a vanishing magnetic field. Switching on a magnetic field induces currents as well as nonzero densities. Again, it is convenient to express the number densities in terms of the quark flavor components, $n_{1,2} \equiv n_{B} \pm n_{I}$, rather than in baryon and isospin components. We obtain for small and large magnetic fields

$$
n_{i}^{\sigma} \simeq \frac{4 \kappa M_{\mathrm{KK}}^{2}}{3} \begin{cases}\frac{\pi q_{i}^{2} \mu_{i} B_{\mathrm{em}}^{2}}{3} & \text { for small } B_{\mathrm{em}} \\ \mu_{i}\left|q_{i}\right| B_{\mathrm{em}} & \text { for large } B_{\mathrm{em}}\end{cases}
$$

For the pion phase we find from the free energy (4.30)

$$
\begin{aligned}
& n_{B}^{\pi}=0, \\
& n_{I}^{\pi}=\frac{16 \kappa M_{\mathrm{KK}}^{2}}{\pi} \mu_{I} .
\end{aligned}
$$

From the baryon and isospin densities we can immediately deduce the electric charge density $n_{Q}=q_{1} n_{1}+q_{2} n_{2}$. The electric charge of the system is relevant for example in the astrophysical context because in a neutron star the overall electric charge has to vanish. Here we simply observe which electric charge is carried by our system for given chemical potentials. For more realistic applications one would have to require charge neutrality and possibly counterbalance the charge of the chiral condensate for instance by the presence of electrons or protons. For the $\sigma$ phase we find $n_{Q}^{\sigma}=0$ for vanishing magnetic fields, as expected. Switching on a magnetic field induces electric charges in the system. For infinitesimally small $B_{\text {em }}$ a straight line $\mu_{B}=-9 \mu_{I} / 7$ appears in the $\mu_{B^{-}}$ $\mu_{I}$ plane dividing the plane into a region with infinitesimally positive (above/right of the line) and negative (below/left of the line) charge. With increasing magnetic field, giving rise to larger charges, the slope of the line slightly decreases and approaches the value $\mu_{B}=-5 \mu_{I} / 3$ asymptotically for large $B_{\mathrm{em}}$. For the pion phase we have $n_{Q}^{\pi}=n_{I}^{\pi}$, which is positive (negative) for positive (negative) isospin chemical potentials and independent of the baryon chemical potential.

We may finally recover the scenario considered in Ref. [50] as a limit of our more general results. In that paper, a vanishing isospin chemical potential, a vanishing baryon

\footnotetext{
${ }^{4}$ This issue is in fact related to the remark below Eq. (4.19): using a modified action as in Ref. 49] which drops certain surface terms would allow us to use the more natural boundary values $\mu_{B, I}$. We have checked that, apart from the difference in the axial current, the use of the modified action does not lead to qualitative changes in our following results. More precisely, changing the action by hand as in Ref. 49] amounts to multiplying the Chern-Simons action by $3 / 2$, and rescaling the chemical potential by a factor $1 / 2$. For the phase diagram presented in Sec. 4.4, only the latter modification is essential and leads to corresponding quantitative changes.
} 
component of the magnetic field, and an isospin magnetic field constant in the holographic coordinate $z$ (as in Eq. (4.22) ) was considered. For a comparison it is thus instructive to compute the energy density $\epsilon$ of the sigma phase. We write the free energy (4.13) as $\Omega=\epsilon-\mu_{B} n_{B}-\mu_{I} n_{I}$ with $n_{B}, n_{I}$ given in Eqs. (4.33). Then we can express the energy density in terms of the number densities,

$$
\epsilon_{\sigma}=\frac{3}{32 \kappa M_{\mathrm{KK}}^{2}}\left[\left(n_{B}^{\sigma}+n_{I}^{\sigma}\right)^{2} \frac{\rho_{+}}{(\hat{B}+B)^{2}}+\left(n_{B}^{\sigma}-n_{I}^{\sigma}\right)^{2} \frac{\rho_{-}}{(\hat{B}-B)^{2}}\right] .
$$

For small and large magnetic fields we obtain

$$
\epsilon_{\sigma} \simeq\left\{\begin{array}{cl}
\frac{2 \lambda M_{\mathrm{KK}}^{2}}{3 N_{c}}\left[\frac{\left(n_{B}^{\sigma}+n_{I}^{\sigma}\right)^{2}}{(\hat{\mathcal{B}}+\mathcal{B})^{2}}+\frac{\left(n_{B}^{\sigma}-n_{I}^{\sigma}\right)^{2}}{(\hat{\mathcal{B}}-\mathcal{B})^{2}}\right] & \text { for small } \hat{\mathcal{B}}, \mathcal{B} \\
\frac{3 \pi^{2}}{N_{c}}\left[\frac{\left(n_{B}^{\sigma}+n_{I}^{\sigma}\right)^{2}}{|\hat{\mathcal{B}}+\mathcal{B}|}+\frac{\left(n_{B}^{\sigma}-n_{I}^{\sigma}\right)^{2}}{|\hat{\mathcal{B}}-\mathcal{B}|}\right] & \text { for large } \hat{\mathcal{B}, \mathcal{B}},
\end{array}\right.
$$

where we have reinstated the dimensionful magnetic fields according to Eq. (4.10). For large magnetic fields we thus obtain, up to a numerical prefactor, an equation of state as for a free fermion gas in a magnetic field: setting $n_{I}^{\sigma}=\hat{\mathcal{B}}=0$ we have $\epsilon_{\sigma}=6 \pi^{2}\left(n_{B}^{\sigma}\right)^{2} /\left(\mathcal{B} N_{c}\right)$ while for a free gas $\epsilon_{0}=\pi^{2} n_{B}^{2} /\left(\mathcal{B} N_{c}\right)$ [50]. In Ref. [50] even the prefactor is exactly that of the free gas. We have checked that this discrepancy comes from the surface term in the Chern-Simons action: had we dropped the contribution of the surface term (3.11d) for the free energy, we would have reproduced Eqs. (34) and (35) of Ref. [50] exactly (upon setting $n_{I}^{\sigma}=\hat{\mathcal{B}}=0$ in our result). We can also explain this discrepancy in another way. We have computed the free energy, taking into account the Yang-Mills and ChernSimons contributions, and then computed the baryon density (and isospin density) by the thermodynamic relation (4.32). Given this definition of $n_{B}$ we can write

$$
n_{B}=\frac{N_{c}}{6 \pi^{2}}(\hat{\jmath} \hat{\mathcal{B}}+\jmath \mathcal{B})=\frac{N_{c}}{96 \pi^{2}} \int_{-\infty}^{\infty} d z \epsilon_{M N P Q} \operatorname{Tr}\left[\mathcal{F}_{M N} \mathcal{F}_{P Q}\right]
$$

where $M, N, P, Q=z, 1,2,3$, where the first equality can be read off from Eq. (4.13) upon reinstating the dimensionful magnetic field, and the second equality follows from the field strengths in Eqs. (B.9). The resulting expression on the right-hand side differs by a factor $1 / 3$ from the one in Ref. [50]; the latter is normalized such that the baryon number is equal to the instanton number for a static instanton configuration [42] (in the absence of a magnetic field). Consequently, had we required this normalization we would have had to adjust the baryon chemical potential to be $3 \mu_{B}$ with $\mu_{B}$ the baryon chemical potential used above. In this case we would have reproduced the small $B$ limit for the energy density of Ref. [50] exactly. However, in the large $B$ limit we would then have obtained $\epsilon_{\sigma}=2 \epsilon_{0} / 3$. The reason is that, due to the surface term, the functional dependence of the energy density on the magnetic field differs from Ref. [50], deviating by different factors in the two limits of small and large magnetic fields. 


\subsection{Phase diagram and critical magnetic field}

In this section we determine which of the two phases is favored for given values of the chemical potentials and the magnetic field. To this end, notice that the holographic description of our system is our "microscopic" theory; therefore, we have identified the boundary values of the bulk field strengths as $\mathcal{B}_{\mathrm{em}}$, not as $\mathcal{H}_{\mathrm{em}}$. We are interested, however, in a free energy comparison at a fixed external magnetic field $\mathcal{H}_{\mathrm{em}}$. Consequently, we have to apply a Legendre transformation to construct the Gibbs free energy $G$. (See for instance Ref. 15 for an analogous construction.) In the case of the charged pion condensate, where $\mathcal{B}_{\mathrm{em}}=0$, the Gibbs free energy is identical to the above computed free energy,

$$
G_{\pi}=\Omega_{\pi}=-\frac{8 \kappa M_{\mathrm{KK}}^{2}}{\pi} \mu_{I}^{2}
$$

It is convenient to introduce dimensionless free energies $\omega_{\sigma, \pi}$ via

$$
\Omega_{\sigma, \pi}=\frac{M_{\mathrm{KK}}^{4}}{\alpha^{2}} \kappa \omega_{\sigma, \pi} .
$$

As we shall see below, $\kappa$ is the only parameter of the model on which the structure of the phase diagram depends, see Eq. (4.52). The other constants $M_{\mathrm{KK}}$ and $\alpha$ only set the energy scale. To make this $\kappa$ dependence explicit, we have pulled the dimensionless constant $\kappa$ out of $\omega_{\sigma, \pi}$. The dimensionless free energies are, using Eqs. (4.21) and (4.30),

$$
\begin{aligned}
& \omega_{\pi}=-\frac{8 \tilde{\mu}_{I}^{2}}{\pi}, \\
& \omega_{\sigma}=-\frac{2}{3}\left[\left(\tilde{\mu}_{B}+\tilde{\mu}_{I}\right)^{2} \frac{(\hat{B}+B)^{2}}{\rho_{+}(\hat{B}, B)}+\left(\tilde{\mu}_{B}-\tilde{\mu}_{I}\right)^{2} \frac{(\hat{B}-B)^{2}}{\rho_{-}(\hat{B}, B)}\right],
\end{aligned}
$$

where we have introduced the dimensionless chemical potentials

$$
\tilde{\mu}_{B} \equiv \frac{\alpha \mu_{B}}{M_{\mathrm{KK}}}, \quad \tilde{\mu}_{I} \equiv \frac{\alpha \mu_{I}}{M_{\mathrm{KK}}} .
$$

To obtain the Gibbs free energy in the sigma phase we add the contribution $\mathcal{B}_{\mathrm{em}}^{2} / 2$ and Legendre transform the free energy with respect to the change of variable $\mathcal{B}_{\mathrm{em}} \rightarrow \mathcal{H}_{\mathrm{em}}$. Consequently,

$$
G_{\sigma}=\frac{1}{2} \mathcal{B}_{\mathrm{em}}^{2}+\Omega_{\sigma}-\mathcal{B}_{\mathrm{em}} \mathcal{H}_{\mathrm{em}}
$$

For a given external field $\mathcal{H}_{\mathrm{em}}$ one determines $\mathcal{B}_{\mathrm{em}}$ from the stationarity condition

$$
0=\frac{\partial G_{\sigma}}{\partial \mathcal{B}_{\mathrm{em}}}=\mathcal{B}_{\mathrm{em}}-\mathcal{M}_{\sigma}-\mathcal{H}_{\mathrm{em}}
$$

where we defined the magnetization in the sigma phase

$$
\mathcal{M}_{\sigma}=-\frac{\partial \Omega_{\sigma}}{\partial \mathcal{B}_{\mathrm{em}}}=\frac{M_{\mathrm{KK}}^{2}}{\alpha} \kappa M_{\sigma}
$$

Here, the dimensionless magnetization is given by

$$
M_{\sigma}=\frac{4}{3}\left[q_{1} \tilde{\mu}_{1}^{2} \frac{\hat{B}+B}{\rho_{+}}\left(1-\frac{\hat{B}+B}{2 \rho_{+}} \frac{\partial \rho_{+}}{\partial \hat{B}}\right)+q_{2} \tilde{\mu}_{2}^{2} \frac{\hat{B}-B}{\rho_{-}}\left(1-\frac{\hat{B}-B}{2 \rho_{-}} \frac{\partial \rho_{-}}{\partial \hat{B}}\right)\right],
$$


where we have used Eq. (3.2) for the electromagnetic field, and expressed the derivatives with respect to $B$ through derivatives with respect to $\hat{B}$. Before coming back to the Gibbs free energy let us discuss the magnetization and the resulting magnetic susceptibility $\chi_{\sigma}$. To obtain $M_{\sigma}$ as a function of the external magnetic field we first solve Eq. (4.45), which, in dimensionless quantities reads

$$
H_{\mathrm{em}}=B_{\mathrm{em}}-\kappa M_{\sigma}
$$

numerically for $B_{\mathrm{em}}$. (Here, the dimensionless field $H_{\mathrm{em}}$ is defined analogously to the field $B_{\text {em }}$, see Eq. (4.10).) Then, we insert the solution back into Eq. (4.47). The result depends on $\kappa$, for which we have to choose a numerical value. In order to get some numerical estimates from our following results we also need to assign values to the other parameters of the model. Following [17, 18, 43] we shall use

$$
\kappa=0.00745, \quad M_{\mathrm{KK}}=949 \mathrm{MeV},
$$

which has been obtained from fits to the rho meson mass and the pion decay constant. From this value for $\kappa$ we obtain, with $N_{c}=3$ and Eq. (3.10), the 't Hooft coupling $\lambda \approx 16.6$, and then, with Eq. (3.7), $\alpha \approx 2.55$.

The full numerical result for the magnetization is shown in Fig. 3. Our result is in qualitative agreement with Ref. [49], where the magnetization was computed for a one-flavor system. (Note, however, that in this reference the boundary value of the field strength was interpreted as $H$, not $B$.) We see that the magnetization behaves linearly for small magnetic fields. The slope is the magnetic susceptibility, i.e., $M_{\sigma} \simeq \chi_{\sigma} H_{\mathrm{em}}$. Upon expanding Eq. (4.47) for small magnetic fields we find

$$
\chi_{\sigma}=\frac{2 \pi}{9} \frac{q_{1}^{2} \tilde{\mu}_{1}^{2}+q_{2}^{2} \tilde{\mu}_{2}^{2}}{1-\frac{2 \kappa \pi}{9}\left(q_{1}^{2} \tilde{\mu}_{1}^{2}+q_{2}^{2} \tilde{\mu}_{2}^{2}\right)} .
$$

Since we neither expect the susceptibility to diverge nor to change sign, this result can only be trusted for sufficiently small chemical potentials, roughly speaking $\tilde{\mu}_{i}^{2} \ll 1 /\left(\kappa e^{2}\right)$. Given the numerical value $\left(\kappa e^{2}\right)^{-1 / 2} \simeq 38$ and given that one unit of the quark chemical potential $\tilde{\mu}_{i}=1$ corresponds to $\mu_{i} \simeq 400 \mathrm{MeV}$, this is not a severe restriction for realistic values of $\mu_{i}$. However, this result shows that in principle one has to be careful with large chemical potentials in the present approximation where we not only have expanded the DBI action for small gauge fields but also neglected the backreaction of the branes to the background geometry.

For large magnetic fields the magnetization saturates. From Eq. (4.47) we find the constant value

$$
\lim _{H_{\mathrm{em}} \rightarrow \infty} M_{\sigma}=\frac{q_{1} \tilde{\mu}_{1}^{2}-q_{2} \tilde{\mu}_{2}^{2}}{3},
$$

where we have used that $B>\hat{B}$ for a two-flavor system with up and down quarks.

We now return to the free energy comparison. For the sigma phase we insert Eq. (4.45) into the Gibbs free energy (4.44). Then we obtain the following difference in Gibbs free energies

$$
\frac{\Delta G}{M_{\mathrm{KK}}^{4} / \alpha^{2}} \equiv \frac{G_{\sigma}-G_{\pi}}{M_{\mathrm{KK}}^{4} / \alpha^{2}}=-\frac{1}{2} H_{\mathrm{em}}^{2}+\frac{1}{2} \kappa^{2} M_{\sigma}^{2}+\kappa\left(\omega_{\sigma}-\omega_{\pi}\right) \text {. }
$$




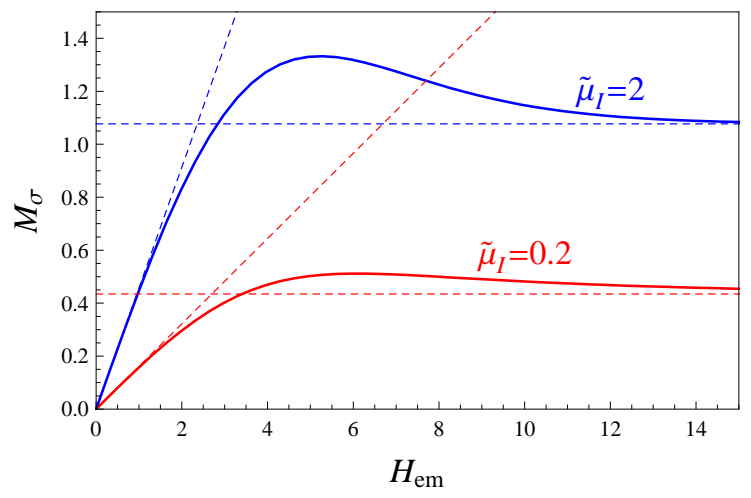

Figure 3: Dimensionless magnetization $M_{\sigma}$ for the sigma phase as a function of the dimensionless magnetic field $H_{\mathrm{em}}$ for two different values of the isospin chemical potential and a baryon chemical potential $\tilde{\mu}_{B}=2$. The dashed lines are the susceptibilities $\chi_{\sigma}$ from Eq. (4.50), which approximate the magnetization for small magnetic fields, $M_{\sigma}=\chi_{\sigma} H_{\mathrm{em}}$, and the asymptotic values from Eq. (4.51).

If $\Delta G>0(\Delta G<0)$ the $\pi(\sigma)$ phase is preferred. It is instructive to relate the comparison between the sigma and pion phases to a usual superconducting material, say a metal, where we compare the superconducting phase (corresponding to the pion phase) and the normalconducting phase (corresponding to the sigma phase). With the help of this analogy we can understand the various terms in $\Delta G$. The term quadratic in $H_{\mathrm{em}}$ is negative, i.e., it works in favor of the normal-conducting phase. This term is the free energy cost which the superconducting phase has to pay for creating a counter magnetic field in order to expel the external magnetic field. In a usual superconductor, this term is thus responsible for a critical magnetic field beyond which the Cooper pair condensate breaks down. There is an additional term, working against the normal-conducting phase, proportional to $M_{\sigma}^{2}$. This term is absent in most usual superconductors which, to a good approximation, have no magnetic properties in their normal-conducting phase. We thus expect a competition between the two terms, i.e., between the costs that the sigma and charged pion phase have to pay for the magnetization and the Meissner effect, respectively. This competition, together with the difference $\omega_{\sigma}-\omega_{\pi}$, will determine the resulting phase diagram.

For small magnetic fields, $H_{\mathrm{em}} \ll 1$, and dimensionless chemical potentials of order one, $\tilde{\mu}_{B, I} \lesssim 1$, we can discuss the phase transition between the sigma and the charged pion phase analytically. In this case, the term $\kappa^{2} M_{\sigma}^{2}$ is of the order of $\kappa^{2} e^{4}$ and thus negligible. The free energy of the sigma phase $\kappa \omega_{\sigma}$ is proportional to $\kappa e^{2}$ and thus also small compared to the remaining terms. We are left with the simple result

$$
\frac{\Delta G}{M_{\mathrm{KK}}^{4} / \alpha^{2}} \simeq-\frac{1}{2} H_{\mathrm{em}}^{2}+\frac{8 \kappa}{\pi} \tilde{\mu}_{I}^{2}
$$

At the phase transition $\Delta G=0$ we thus have

$$
\tilde{\mu}_{I}= \pm \sqrt{\frac{\pi}{\kappa}} \frac{H_{\mathrm{em}}}{4} \approx \pm 5.13 H_{\mathrm{em}} .
$$




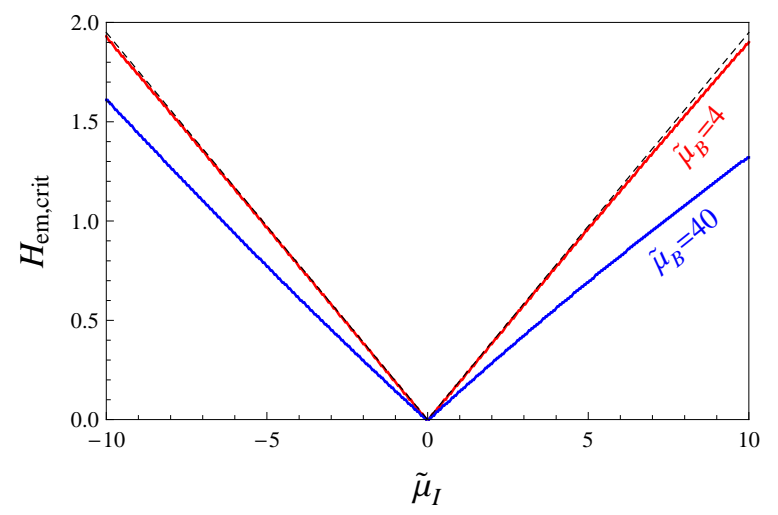

Figure 4: Critical magnetic field for the phase transition from the pion to the sigma phase as a function of the isospin chemical potential for baryon chemical potentials $\tilde{\mu}_{B}=4$ (red curve) and $\tilde{\mu}_{B}=40$ (blue curve). The dashed line is the approximation from Eq. (4.54) and almost coincides with the curve for $\tilde{\mu}_{B}=4$. Our model does not include a finite pion mass. It can be expected that the effect of the pion mass shifts the curves such that they start at $\mu_{I}= \pm m_{\pi}$ (corresponding to $\tilde{\mu}_{I} \simeq \pm 0.375$ with the choice (4.49)) instead of at $\mu_{I}=0$.

This relation can be read as an equation for the critical magnetic field for a given isospin chemical potential, or as an equation for the critical chemical potential for a given magnetic field. We see that, in this approximation, the phase transition is independent of the baryon chemical potential. In Fig. $⿴$ we plot the critical magnetic field as a function of $\mu_{I}$ for two different values of $\mu_{B}$.

Let us now discuss the resulting phase diagram in the $\mu_{B}-\mu_{I}$ plane. Firstly, we consider the case of a vanishing magnetic field, $H_{\mathrm{em}}=0$. From Eq. (4.53) we see that, in this case, the pion phase is favored in the entire $\mu_{B}-\mu_{I}$ plane except for the $\mu_{B}$ axis. To understand this result we recall several features of our model. We treat the fermions as massless (in most applications of the Sakai-Sugimoto model, this approximation is used; for a discussion about how to incorporate finite mass effects into the model see Ref. 850). Therefore, a charged pion condensate appears for arbitrarily small isospin chemical potentials and not only beyond a finite threshold given by the pion mass. Moreover, since we consider the confined phase, we cannot account for phases where there is a vanishing $\langle\bar{u} u\rangle$ and a nonvanishing $\langle\bar{d} d\rangle$ condensate or vice versa. In other words, we cannot connect the upflavor branes and leave the down-flavor branes disconnected, as done in Ref. [65], where the deconfined (but chirally broken) phase was considered. And finally, in our approach we do not see a phase transition to the chirally restored phase. Since we are in the confined phase, where the subspace of the compactified extra dimension $x_{4}$ and the holographic coordinate $z$ is cigar-shaped, the $\mathrm{D} 8$ and $\overline{\mathrm{D} 8}$ branes must connect, i.e., chiral symmetry must be broken for all values of the chemical potentials. The chiral symmetry can only be restored above the deconfinement phase transition at $T_{c}=M_{\mathrm{KK}} /(2 \pi)$, i.e., using (4.49), $T_{c} \approx 150 \mathrm{MeV}$. Taking into account these restrictions, our phase diagram at vanishing magnetic field is in accordance for instance with Refs. [65, 79, 86]. 


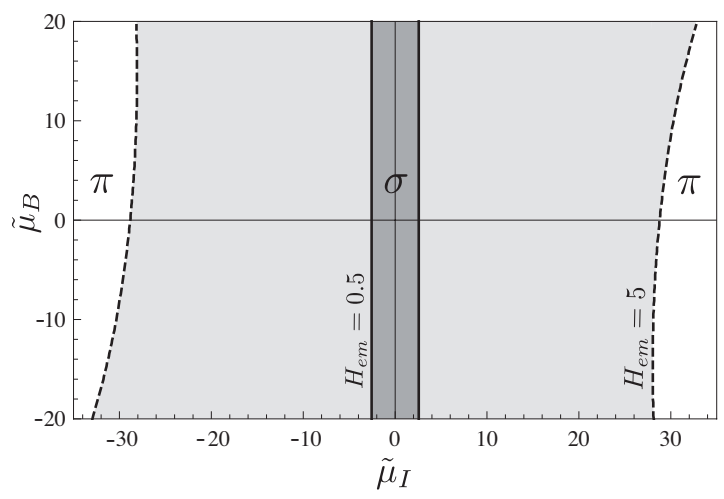

Figure 5: Phase diagram for the $\sigma$ and $\pi$ phases in the plane of baryon and isospin chemical potentials. We have chosen two different values of the dimensionless magnetic field, $H_{\mathrm{em}}=0.5$ (solid lines, dark shaded sigma phase) and $H_{\mathrm{em}}=5$ (dashed lines, light shaded sigma phase). The sigma phase contains meson supercurrents while the pion phase contains an isotropic $\pi^{ \pm}$condensate and exhibits the Meissner effect. All lines indicate first order phase transitions. The units of this plot, upon fitting the parameters of the model according to (4.49), are $\tilde{\mu}_{B, I} \approx \mu_{B, I} /(370 \mathrm{MeV})$ and $H_{\mathrm{em}} \approx \mathcal{H}_{\mathrm{em}} /\left(1.8 \cdot 10^{19} \mathrm{G}\right)$. Hence, due to the huge scales, even compared to magnetar scales, this phase diagram is rather of academic interest; for more realistic chemical potentials and magnetic fields, the simple approximation (4.54) is sufficient.

Next, we discuss the case of a nonzero magnetic field. The phase diagram for two different magnetic fields is shown in Fig. 5. From Eq. (4.54) we see that a region for the sigma phase opens up, with straight phase transition lines independent of $\mu_{B}$. These lines start to bend for larger magnetic fields. We may use the numerical values of the parameters of the model given in Eq. (4.49) and below that equation for some (very) rough quantitative predictions from this phase diagram. First we notice that a dimensionless field $H_{\mathrm{em}}=1$ corresponds to ${ }^{5} \mathcal{H}_{\mathrm{em}} \simeq 2 \cdot 10^{19} \mathrm{G}$, about $4-5$ orders of magnitude larger than the surface field of magnetars, and most likely even larger than the magnetic field in the interior of the star. For the chemical potentials we find that $\tilde{\mu}_{B, I}=1$ corresponds to $\mu_{B, I} \simeq 400 \mathrm{MeV}$. As a comparison, a typical baryon chemical potential for neutron stars is at most $\mu_{B} \lesssim 1500 \mathrm{MeV}$, corresponding to $\tilde{\mu}_{B} \simeq 4$. Now, as a rough estimate, let us assume an isospin chemical potential of $1 / 10$ times the baryon chemical potential in a neutron star, i.e., $\tilde{\mu}_{I} \simeq 0.4$. Then, the phase transition from the charged pion phase to the sigma phase occurs at a very large magnetic field of approximately $\mathcal{H}_{\mathrm{em}} \simeq 1.6 \cdot 10^{18} \mathrm{G}$. In other words, the charged pion condensate at finite isospin chemical potentials appears to be, in terms of realistic values for the magnetic field, very robust.

The superconducting properties of a charged pion condensate have been studied in conventional chiral models 87, 88, 89, 90. There, for an isotropic charged pion condensate, the scale of the critical magnetic field is set by $m_{\pi}^{2}$, which is of the order of $10^{18} \mathrm{G}$, whereas an anisotropic charged pion condensate has been argued to give a critical magnetic field of

\footnotetext{
${ }^{5}$ In the natural Heavyside-Lorentz system of units of particle physics, a magnetic field strength of $1 \mathrm{eV}^{2}$ corresponds to $51.189 . . \mathrm{G}$ in the Gaussian system.
} 
the order of $10^{19} \mathrm{G}$. In our model, in the $\pi$ phase we have an isotropic $\pi$ condensate and a vanishing $m_{\pi}$ (see in this context Ref. [91]), but nevertheless we have obtained a critical field of comparable magnitude. It should be noted that in conventional chiral models the charged pion condensate has been found to behave as a type-II superconductor [87, 88, 89], which means that there is another, smaller critical field strength, above which the magnetic field can penetrate in the form of magnetic flux tubes. By considering only homogeneous fields, we have of course not taken this possibility into account.

\section{Summary and outlook}

We have employed the Sakai-Sugimoto model to study chiral symmetry breaking in a two-flavor system with a magnetic field and baryon and isospin chemical potentials. The model consists of a D4/D8- $\overline{\mathrm{D} 8}$ system in which a bulk gauge symmetry on the D8 and $\overline{\mathrm{D} 8}$ branes corresponds to a global (flavor) symmetry at the boundary which is interpreted as chiral symmetry. Here, left- and right-handed fermions are separated by a fifth extra dimension. The electromagnetic subgroup of this flavor symmetry group has been used to incorporate an external magnetic field. Our results are independent of temperature, valid for temperatures below the critical temperature for chiral symmetry breaking. We have discussed how the model can account for different Goldstone boson condensates, two of which we have described within our ansatz of abelian gauge field components. Starting from the D-brane action, consisting of Yang-Mills plus Chern-Simons contributions, we have solved the equations of motion analytically and computed the Gibbs free energies of these two phases.

The first phase, briefly termed sigma phase, is a linear combination of the usual chiral sigma condensate and a neutral pion condensate. We have found that for nonzero magnetic fields this phase exhibits nonzero meson supercurrents. In addition, normal currents are generated. For both types of currents, there exist counter-propagating currents such that the net vector current of the system is zero. This is reminiscent of unconventional (anisotropic) superfluids and superconductors in condensed matter systems or deconfined dense quark matter. We have found that the baryon and isospin densities in the system, which obviously vanish without a magnetic field, become nonzero once a magnetic field is switched on. As a consequence, also an electric charge appears in the sigma phase.

In the second phase, briefly termed pion phase, charged pions form a condensate. This phase reacts very differently to a magnetic field. It acts as an electromagnetic superconductor, and thus expels the magnetic field due to the Meissner effect. We have seen that the assumption of a nonzero magnetic field would lead to infrared divergences in the energy density which cannot be removed by holographic renormalization. Therefore, we have introduced supercurrents which induce a magnetic field opposite, but equal in magnitude, to the externally applied field. Then the total magnetic field in the $\pi$ phase vanishes, and a consistent treatment without divergences is possible. In contrast to the $\sigma$ phase, the $\pi$ phase, due to the Meissner effect, appears unaltered under the influence of a magnetic field. In particular, the baryon number and all currents (except for the supercurrents cancelling the external magnetic field) vanish. 
Besides the calculation of the supercurrents and the observation of the Meissner effect in the charged $\pi$ condensate, the main result of our work is the free energy comparison between the two phases and the resulting phase diagram in the $\mu_{B^{-}} \mu_{I}$ plane. For a vanishing magnetic field, a nonzero isospin chemical potential leads to the rotation of the sigma condensate into a charged pion condensate. This is expected from studies using the same and other models [65, 77, 79, 91, 92]. In the present study, which does not include quark masses, this means that in the absence of a magnetic field the pion condensate is favored over the sigma condensate in the entire $\mu_{B^{-}} \mu_{I}$ plane. For a nonzero magnetic field the rotation is partially undone, i.e., for a given external magnetic field, there is a region for sufficiently small $\mu_{I}$ where the $\sigma$ phase is favored over the $\pi$ phase. This is not unlike the transition in a metal from its superconducting to its normal conducting state. We have found that for small magnetic fields, the critical magnetic field for this phase transition is linear in $\mu_{I}$ and independent of $\mu_{B}, H_{c} \propto\left|\mu_{I}\right|$. As a quantitative estimate from our result we have discussed that for magnetic fields on compact star scales, the charged pion phase at nonzero $\mu_{I}$ is very robust. More precisely, magnetic fields of the order of $10^{18} \mathrm{G}$ (well beyond surface magnetic fields of magnetars, although conceivable for their interiors) are needed to induce a phase transition from the $\pi$ to the $\sigma$ phase for isospin chemical potentials of the order of $150 \mathrm{MeV}$.

There are several interesting extensions to our work. One could consider the case of not maximally separated $\mathrm{D} 8$ and $\overline{\mathrm{D} 8}$ branes. This would allow for the possibility of the chirally restored phase even at small temperatures and thus might answer the question of how a magnetic field affects this symmetry restoration. This extension requires to solve an additional equation of motion for the geometry of the $\mathrm{D} 8$ and $\overline{\mathrm{D} 8}$ branes, probably making an analytical solution impossible. One may also study the question whether the solutions found here (anisotropic but homogeneous) are stable against formation of crystalline structures. Moreover, since charged pion condensates have been found to behave as type-II superconductors in conventional chiral models [87, 88, 89], it would be interesting to consider inhomogeneous vortex-like configurations of magnetic fields. It is also important to check whether the states we have described are stable with respect to other meson condensates. We already know that without magnetic field a rho meson condensate is expected to form for sufficiently large isospin chemical potentials [66]. Another problem, not directly related to the present study, but intimately related to the interplay of chirality

and a magnetic field, is the chiral magnetic effect [93]. It seems that the Sakai-Sugimoto model provides all ingredients to study this effect.

\section{Acknowledgments}

We thank M. Alford, D. Grumiller, E. Fraga, C. Herzog, M. Kaminski, K. Landsteiner, G. Lifschytz, E. Lopez, K. Peeters, R. Rashkov, D.T. Son, and A. Vuorinen for valuable comments and discussions and acknowledge support by the FWF projects P19526, P19958. 


\section{A. General form of equations of motion}

Here we present the general form of the variations of the Yang-Mills Lagrangian $\mathcal{L}_{\mathrm{YM}}$ and

the Chern-Simons Lagrangian $\mathcal{L}_{\mathrm{CS}}$ (which can be read off from Eqs. (2.12) and (2.16) for the chirally broken phase. The variation of the Yang-Mills Lagrangian is

$$
\frac{\delta \mathcal{L}_{\mathrm{YM}}}{\delta \mathcal{A}_{\lambda}^{a}}=-2 T_{8} V_{4}\left(2 \pi \alpha^{\prime}\right)^{2} \operatorname{Tr}\left[\left(t_{a} \partial_{\rho}-i\left[t_{a}, \mathcal{A}_{\rho}\right]\right) e^{-\Phi} \sqrt{g} g^{\mu \lambda} g^{\rho \sigma} \mathcal{F}_{\sigma \mu}\right]
$$

where the greek indices run over $0,1,2,3, u$, and where $t_{0} \equiv \mathbf{1} / 2, t_{a} \equiv \tau_{a} / 2$, according to the convention (2.14). Consequently,

$$
\begin{aligned}
& -\frac{3 u_{\mathrm{KK}}^{3 / 2}}{4 \kappa M_{\mathrm{KK}}^{2}} \frac{\delta \mathcal{L}_{\mathrm{YM}}}{\delta \hat{A}_{0}}=\partial_{u}\left(\frac{u^{5 / 2} f^{1 / 2}}{v} \hat{F}_{u 0}\right)+\partial_{i}\left(\frac{R^{3} v}{u^{1 / 2} f^{1 / 2}} \hat{F}_{i 0}\right) \\
& -\frac{3 u_{\mathrm{KK}}^{3 / 2}}{4 \kappa M_{\mathrm{KK}}^{2}} \frac{\delta \mathcal{L}_{\mathrm{YM}}}{\delta \hat{A}_{i}}=\partial_{u}\left(\frac{u^{5 / 2} f^{1 / 2}}{v} \hat{F}_{u i}\right)+\partial_{0}\left(\frac{R^{3} v}{u^{1 / 2} f^{1 / 2}} \hat{F}_{0 i}\right)+\partial_{j}\left(\frac{R^{3} v}{u^{1 / 2} f^{1 / 2}} \hat{F}_{j i}\right), \\
& -\frac{3 u_{\mathrm{KK}}^{3 / 2}}{4 \kappa M_{\mathrm{KK}}^{2}} \frac{\delta \mathcal{L}_{\mathrm{YM}}}{\delta \hat{A}_{u}}=\partial_{0}\left(\frac{u^{5 / 2} f^{1 / 2}}{v} \hat{F}_{0 u}\right)+\partial_{i}\left(\frac{u^{5 / 2} f^{1 / 2}}{v} \hat{F}_{i u}\right)
\end{aligned}
$$

and

$$
\begin{aligned}
-\frac{3 u_{\mathrm{KK}}^{3 / 2}}{4 \kappa M_{\mathrm{KK}}^{2}} \frac{\delta \mathcal{L}_{\mathrm{YM}}}{\delta A_{0}^{a}}= & \left(\delta_{a c} \partial_{u}+A_{u}^{b} \epsilon_{a b c}\right) \frac{u^{5 / 2} f^{1 / 2}}{v} F_{u 0}^{c}+\left(\delta_{a c} \partial_{i}+A_{i}^{b} \epsilon_{a b c}\right) \frac{R^{3} v}{u^{1 / 2} f^{1 / 2}} F_{i 0}^{c}, \\
-\frac{3 u_{\mathrm{KK}}^{3 / 2}}{4 \kappa M_{\mathrm{KK}}^{2}} \frac{\delta \mathcal{L}_{\mathrm{YM}}}{\delta A_{i}^{a}}= & \left(\delta_{a c} \partial_{u}+A_{u}^{b} \epsilon_{a b c}\right) \frac{u^{5 / 2} f^{1 / 2}}{v} F_{u i}^{c}+\left(\delta_{a c} \partial_{0}+A_{0}^{b} \epsilon_{a b c}\right) \frac{R^{3} v}{u^{1 / 2} f^{1 / 2}} F_{0 i}^{c} \\
& +\left(\delta_{a c} \partial_{j}+A_{j}^{b} \epsilon_{a b c}\right) \frac{R^{3} v}{u^{1 / 2} f^{1 / 2}} F_{j i}^{c}, \\
-\frac{3 u_{\mathrm{KK}}^{3 / 2}}{4 \kappa M_{\mathrm{KK}}^{2}} \frac{\delta \mathcal{L}_{\mathrm{YM}}}{\delta A_{u}^{a}}= & \left(\delta_{a c} \partial_{0}+A_{0}^{b} \epsilon_{a b c}\right) \frac{u^{5 / 2} f^{1 / 2}}{v} F_{0 u}^{c}+\left(\delta_{a c} \partial_{i}+A_{i}^{b} \epsilon_{a b c}\right) \frac{u^{5 / 2} f^{1 / 2}}{v} F_{i u}^{c},
\end{aligned}
$$

where the indices $i, j, k$ run over $1,2,3$, and where we used

$$
\frac{T_{8} V_{4}\left(2 \pi \alpha^{\prime}\right)^{2} R^{3 / 2}}{g_{s}}=\frac{4 \kappa M_{\mathrm{KK}}^{2}}{3 u_{\mathrm{KK}}^{3 / 2}},
$$

with $\kappa$ defined in Eq. (3.10).

The variations of the Chern-Simons Lagrangian with respect to the $\mathbf{1}$ and $\tau_{3}$ components are

$$
\begin{aligned}
& \frac{\delta \mathcal{L}_{\mathrm{CS}}}{\delta \hat{A}_{\mu}}=-i \frac{\kappa \alpha}{4}\left(F_{\nu \rho}^{a} F_{\sigma \lambda}^{a}+\hat{F}_{\nu \rho} \hat{F}_{\sigma \lambda}\right) \epsilon^{\mu \nu \rho \sigma \lambda}, \\
& \frac{\delta \mathcal{L}_{\mathrm{CS}}}{\delta A_{\mu}^{a}}=-i \frac{\kappa \alpha}{2} \hat{F}_{\nu \rho} F_{\sigma \lambda}^{a} \epsilon^{\mu \nu \rho \sigma \lambda}
\end{aligned}
$$


with $\alpha$ defined in Eq. (3.7). Consequently,

$$
\begin{aligned}
& \frac{\delta \mathcal{L}_{\mathrm{CS}}}{\delta \hat{A}_{0}}=i \kappa \alpha\left(F_{u i}^{a} F_{j k}^{a}+\hat{F}_{u i} \hat{F}_{j k}\right) \epsilon^{i j k}, \\
& \frac{\delta \mathcal{L}_{\mathrm{CS}}}{\delta \hat{A}_{i}}=i \kappa \alpha\left(2 F_{j 0}^{a} F_{u k}^{a}-F_{u 0}^{a} F_{j k}^{a}+2 \hat{F}_{j 0} \hat{F}_{u k}-\hat{F}_{u 0} \hat{F}_{j k}\right) \epsilon^{i j k}, \\
& \frac{\delta \mathcal{L}_{\mathrm{CS}}}{\delta \hat{A}_{u}}=i \kappa \alpha\left(F_{i 0}^{a} F_{j k}^{a}+\hat{F}_{i 0} \hat{F}_{j k}\right) \epsilon^{i j k},
\end{aligned}
$$

and

$$
\begin{aligned}
& \frac{\delta \mathcal{L}_{\mathrm{CS}}}{\delta A_{0}^{a}}=i \kappa \alpha\left(F_{u i}^{a} \hat{F}_{j k}+F_{j k}^{a} \hat{F}_{u i}\right) \epsilon^{i j k}, \\
& \frac{\delta \mathcal{L}_{\mathrm{CS}}}{\delta A_{i}^{a}}=i \kappa \alpha\left(2 F_{u k}^{a} \hat{F}_{j 0}-F_{j k}^{a} \hat{F}_{u 0}+2 F_{j 0}^{a} \hat{F}_{u k}-F_{u 0}^{a} \hat{F}_{j k}\right) \epsilon^{i j k}, \\
& \frac{\delta \mathcal{L}_{\mathrm{CS}}}{\delta A_{u}^{a}}=i \kappa \alpha\left(F_{i 0}^{a} \hat{F}_{j k}+F_{j k}^{a} \hat{F}_{i 0}\right) \epsilon^{i j k} .
\end{aligned}
$$

As mentioned in the main part of the paper, we consider maximally separated branes $L=\pi / M_{\mathrm{KK}}$, for which the embedding of the $\mathrm{D} 8$ branes is trivial, $\partial_{u} x_{4}=0$, and thus $v=1$ (see Eq. (2.10)). This simplifies the above expressions and also ensures that there is no equation of motion for $x_{4}(u)$. The expressions A.2), (A.3), (A.6), (A.7) are used in Sec. 3.1 to derive the field equations for our specific ansatz.

\section{B. Solving the equations of motion for constant magnetic fields}

In this appendix we solve the equations of motion for a constant magnetic field for general boundary conditions. The resulting general expressions are instructive to see the structure and symmetries of the solution. By inserting the boundary conditions from Table 1 into the general expressions we obtain the solution for the sigma phase, see Eqs. (4.11) (the pion phase requires a nonconstant magnetic field and is discussed in Appendix Q). The general boundary conditions used here are denoted by

$$
\begin{array}{ll}
\hat{A}_{0}( \pm \infty)=2 \mu_{B}^{R, L}, & A_{0}( \pm \infty)=2 \mu_{I}^{R, L}, \\
\hat{A}_{3}( \pm \infty)=2 \hat{\jmath}^{R, L}, & A_{3}( \pm \infty)=2 \jmath^{R, L},
\end{array}
$$

where the upper (lower) sign corresponds to $R(L)$. It is convenient to express the boundary values in terms of their sums and differences,

$$
\begin{aligned}
\mu_{B, I}^{V} & \equiv \frac{\mu_{B, I}^{R}+\mu_{B, I}^{L}}{2}, \quad \mu_{B, I}^{A} \equiv \frac{\mu_{B, I}^{R}-\mu_{B, I}^{L}}{2}, \\
\hat{J} & \equiv \frac{\hat{\jmath}^{R}+\hat{\jmath}^{L}}{2}, \quad \hat{\jmath} \equiv \frac{\hat{\jmath}^{R}-\hat{\jmath}^{L}}{2}, \quad J \equiv \frac{\jmath^{R}+\jmath^{L}}{2}, \quad \jmath \equiv \frac{\jmath^{R}-\jmath^{L}}{2} .
\end{aligned}
$$

Here, $V$ and $A$ stand for the vector and axial parts of the chemical potentials.

The general solution for (4.8) with the magnetic field (4.9) is

$$
\begin{aligned}
& F_{0}^{+}=c_{1} \zeta_{+}^{-1}+c_{2} \zeta_{+}, \quad F_{0}^{-}=d_{1} \zeta_{-}^{-1}+d_{2} \zeta_{-}, \\
& F_{3}^{+}=-c_{1} \zeta_{+}^{-1}+c_{2} \zeta_{+}, \quad F_{3}^{-}=-d_{1} \zeta_{-}^{-1}+d_{2} \zeta_{-},
\end{aligned}
$$


with constants $c_{1}, c_{2}, d_{1}, d_{2}$ and with

$$
\zeta_{ \pm}(z) \equiv e^{(\hat{B} \pm B) \arctan z}
$$

Consequently, from Eqs. (4.7) we obtain

$$
\begin{aligned}
& k \hat{F}_{z 0}=c_{1} \zeta_{+}^{-1}+c_{2} \zeta_{+}+d_{1} \zeta_{-}^{-1}+d_{2} \zeta_{-}, \\
& k F_{z 0}=c_{1} \zeta_{+}^{-1}+c_{2} \zeta_{+}-d_{1} \zeta_{-}^{-1}-d_{2} \zeta_{-}, \\
& k \hat{F}_{z 3}=-c_{1} \zeta_{+}^{-1}+c_{2} \zeta_{+}-d_{1} \zeta_{-}^{-1}+d_{2} \zeta_{-} \\
& k F_{z 3}=-c_{1} \zeta_{+}^{-1}+c_{2} \zeta_{+}+d_{1} \zeta_{-}^{-1}-d_{2} \zeta_{-}
\end{aligned}
$$

Here and in the remainder of this and the following appendices we often omit the argument $z$ in the various functions for the sake of brevity. For the integration of the field strengths we use

$$
\int d z \frac{\zeta_{ \pm}(z)}{k(z)}=\frac{\zeta_{ \pm}(z)}{\hat{B} \pm B}, \quad \int d z \frac{\zeta_{ \pm}^{-1}(z)}{k(z)}=-\frac{\zeta_{ \pm}^{-1}(z)}{\hat{B} \pm B}
$$

This yields the gauge fields

$$
\begin{aligned}
& \hat{A}_{0}=-\frac{c_{1} \zeta_{+}^{-1}}{\hat{B}+B}+\frac{c_{2} \zeta_{+}}{\hat{B}+B}-\frac{d_{1} \zeta_{-}^{-1}}{\hat{B}-B}+\frac{d_{2} \zeta_{-}}{\hat{B}-B}+\hat{a}_{0}, \\
& A_{0}=-\frac{c_{1} \zeta_{+}^{-1}}{\hat{B}+B}+\frac{c_{2} \zeta_{+}}{\hat{B}+B}+\frac{d_{1} \zeta_{-}^{-1}}{\hat{B}-B}-\frac{d_{2} \zeta_{-}}{\hat{B}-B}+a_{0}, \\
& \hat{A}_{3}=\frac{c_{1} \zeta_{+}^{-1}}{\hat{B}+B}+\frac{c_{2} \zeta_{+}}{\hat{B}+B}+\frac{d_{1} \zeta_{-}^{-1}}{\hat{B}-B}+\frac{d_{2} \zeta_{-}}{\hat{B}-B}+\hat{a}_{3}, \\
& A_{3}=\frac{c_{1} \zeta_{+}^{-1}}{\hat{B}+B}+\frac{c_{2} \zeta_{+}}{\hat{B}+B}-\frac{d_{1} \zeta_{-}^{-1}}{\hat{B}-B}-\frac{d_{2} \zeta_{-}}{\hat{B}-B}+a_{3},
\end{aligned}
$$

with integration constants $\hat{a}_{0}, a_{0}, \hat{a}_{3}, a_{3}$. We determine the eight constants from the eight boundary conditions (B.1). This yields the gauge fields

$$
\begin{aligned}
\hat{A}_{0}= & 2 \mu_{B}^{V}+\mu_{B}^{A}\left(S_{+}+S_{-}\right)+\mu_{I}^{A}\left(S_{+}-S_{-}\right) \\
& +\hat{\jmath}\left(C_{+}+C_{-}-T_{+}\right)+\jmath\left(C_{+}-C_{-}-T_{-}\right), \\
A_{0}= & 2 \mu_{I}^{V}+\mu_{I}^{A}\left(S_{+}+S_{-}\right)+\mu_{B}^{A}\left(S_{+}-S_{-}\right) \\
& +\jmath\left(C_{+}+C_{-}-T_{+}\right)+\hat{\jmath}\left(C_{+}-C_{-}-T_{-}\right), \\
\hat{A}_{3}= & 2 \hat{J}+\hat{\jmath}\left(S_{+}+S_{-}\right)+\jmath\left(S_{+}-S_{-}\right) \\
& +\mu_{B}^{A}\left(C_{+}+C_{-}-T_{+}\right)+\mu_{I}^{A}\left(C_{+}-C_{-}-T_{-}\right), \\
A_{3}= & 2 J+\jmath\left(S_{+}+S_{-}\right)+\hat{\jmath}\left(S_{+}-S_{-}\right) \\
& +\mu_{I}^{A}\left(C_{+}+C_{-}-T_{+}\right)+\mu_{B}^{A}\left(C_{+}-C_{-}-T_{-}\right),
\end{aligned}
$$


and $(k(z)$ times) the field strengths

$$
\begin{aligned}
k \hat{F}_{z 0}= & \mu_{B}^{A}\left[\hat{B}\left(C_{+}+C_{-}\right)+B\left(C_{+}-C_{-}\right)\right]+\mu_{I}^{A}\left[\hat{B}\left(C_{+}-C_{-}\right)+B\left(C_{+}+C_{-}\right)\right] \\
& +\hat{\jmath}\left[\hat{B}\left(S_{+}+S_{-}\right)+B\left(S_{+}-S_{-}\right)\right]+\jmath\left[\hat{B}\left(S_{+}-S_{-}\right)+B\left(S_{+}+S_{-}\right)\right], \\
k F_{z 0}= & \mu_{B}^{A}\left[\hat{B}\left(C_{+}-C_{-}\right)+B\left(C_{+}+C_{-}\right)\right]+\mu_{I}^{A}\left[\hat{B}\left(C_{+}+C_{-}\right)+B\left(C_{+}-C_{-}\right)\right] \\
& +\hat{\jmath}\left[\hat{B}\left(S_{+}-S_{-}\right)+B\left(S_{+}+S_{-}\right)\right]+\jmath\left[\hat{B}\left(S_{+}+S_{-}\right)+B\left(S_{+}-S_{-}\right)\right], \\
k \hat{F}_{z 3}= & \hat{\jmath}\left[\hat{B}\left(C_{+}+C_{-}\right)+B\left(C_{+}-C_{-}\right)\right]+\jmath\left[\hat{B}\left(C_{+}-C_{-}\right)+B\left(C_{+}+C_{-}\right)\right] \\
& +\mu_{B}^{A}\left[\hat{B}\left(S_{+}+S_{-}\right)+B\left(S_{+}-S_{-}\right)\right]+\mu_{I}^{A}\left[\hat{B}\left(S_{+}-S_{-}\right)+B\left(S_{+}+S_{-}\right)\right], \\
k F_{z 3}= & \hat{\jmath}\left[\hat{B}\left(C_{+}-C_{-}\right)+B\left(C_{+}+C_{-}\right)\right]+\jmath\left[\hat{B}\left(C_{+}+C_{-}\right)+B\left(C_{+}-C_{-}\right)\right] \\
& +\mu_{B}^{A}\left[\hat{B}\left(S_{+}-S_{-}\right)+B\left(S_{+}+S_{-}\right)\right]+\mu_{I}^{A}\left[\hat{B}\left(S_{+}+S_{-}\right)+B\left(S_{+}-S_{-}\right)\right],
\end{aligned}
$$

where the functions $C_{ \pm}(z), S_{ \pm}(z)$, and $T_{ \pm}$are defined in Eqs. (4.12). As it should be, the gauge fields (B.8) transform as a vector under a parity transformation. This can be seen as follows. A parity transformation is given by $\left(x_{1}, x_{2}, x_{3}, z\right) \rightarrow\left(-x_{1},-x_{2},-x_{3},-z\right)$. In particular, the transformation $z \rightarrow-z$ implies a chirality transformation $R \rightarrow L$ since the two halves of the $\mathrm{D} 8 / \overline{\mathrm{D} 8}$ branes, namely $z>0$ and $z<0$, correspond to right- and left-handed fermions. Consequently, a parity transformation acts as $C_{ \pm}(z) \rightarrow+C_{ \pm}(z)$, $S_{ \pm}(z) \rightarrow-S_{ \pm}(z)$ (since the magnetic fields $\hat{B}, B$ are even under parity). For the supercurrents we have $\hat{\jmath}, \jmath \rightarrow+\hat{\jmath},+\jmath$ and $\hat{J}, J \rightarrow-\hat{J},-J$. Here we have used that the Goldstone bosons are pseudoscalars (for a detailed discussion of the parity of the mesons in the SakaiSugimoto model see Ref. [17]). As a result we see that the temporal components (B.8a), (B.8b) have even parity, while the spatial components $(\overline{B .8 \mathrm{~d}}),(\mathrm{B} .8 \mathrm{~d})$ have odd parity. This statement is true for arbitrary values of the currents $\hat{\jmath}, \jmath, \hat{J}, J$. We shall see below that in the case of a charged pion condensate the requirement of a well-defined parity results in conditions for the supercurrents, see discussion below Eq. (C.10).

In order to compute the free energy we note that

$$
C_{ \pm}^{2}-S_{ \pm}^{2}=\frac{1}{\sinh ^{2}[\pi(\hat{B} \pm B) / 2]}
$$

Therefore, the following combination of field strengths, needed for the free energy, becomes independent of $z$,

$$
\begin{gathered}
k^{2}\left(-\hat{F}_{z 0}^{2}-F_{z 0}^{2}+\hat{F}_{z 3}^{2}+F_{z 3}^{2}\right)=2\left[(\hat{\jmath}+\jmath)^{2}-\left(\mu_{B}^{A}+\mu_{I}^{A}\right)^{2}\right] \frac{(\hat{B}+B)^{2}}{\sinh ^{2}[\pi(\hat{B}+B) / 2]} \\
+2\left[(\hat{\jmath}-\jmath)^{2}-\left(\mu_{B}^{A}-\mu_{I}^{A}\right)^{2}\right] \frac{(\hat{B}-B)^{2}}{\sinh ^{2}[\pi(\hat{B}-B) / 2]} .
\end{gathered}
$$

Next we use the fact that $S_{ \pm}$and $C_{ \pm}$are antisymmetric and symmetric in $z$, respectively, as well as

$$
C_{ \pm}(\infty)=\operatorname{coth} \frac{\pi(\hat{B} \pm B)}{2}, \quad S_{ \pm}(\infty)=1
$$




\begin{tabular}{|c||c||c|c|}
\hline \multicolumn{1}{|c||}{} & \multicolumn{1}{c||}{ small $\hat{B}, B$} & \multicolumn{2}{c|}{ large $|\hat{B}|,|B|$} \\
\hline & & $|\hat{B}|>|B|$ & $|\hat{B}|<|B|$ \\
\hline \hline$\rho_{ \pm}$ & $\frac{6}{\pi}+\frac{\pi(\hat{B} \pm B)^{2}}{6}$ & \multicolumn{2}{|c|}{$2|\hat{B} \pm B|$} \\
\hline$\rho$ & $\frac{12}{\pi}+\frac{5 \hat{B}^{2}+B^{2}}{15} \pi$ & $4|\hat{B}|$ & $2(|\hat{B}|+|B|)$ \\
\hline$\eta_{+}$ & $\frac{\pi \hat{B} B}{3}$ & $2 B \operatorname{sgn} \hat{B}$ & $B \operatorname{sgn} \hat{B}+\hat{B} \operatorname{sgn} B$ \\
\hline$\eta_{-}$ & $2 \hat{B}$ & $2 \hat{B}$ & $(|\hat{B}|+|B|) \operatorname{sgn} \hat{B}$ \\
\hline
\end{tabular}

Table 2: Behavior of the functions $\rho, \rho_{ \pm}, \eta_{ \pm}$, defined in Eqs. (4.14), (4.27) for small and large magnetic fields $\hat{B}, B$. We have kept relative magnitude and sign of baryon and isospin components arbitrary. They can then later be inserted according to the electric charges of the quarks. We show the behavior for small magnetic fields up to second order and the behavior for large magnetic fields in leading linear order.

to obtain

$$
\begin{aligned}
& \left(\hat{A}_{0} k \hat{F}_{z 0}+A_{0} k F_{z 0}-\hat{A}_{3} k \hat{F}_{z 3}-A_{3} k F_{z 3}\right)_{z=-\infty}^{z=\infty} \\
& =8 \mu_{B}^{V}(\hat{\jmath} \hat{B}+\jmath B)+8 \mu_{I}^{V}(\hat{\jmath} B+\jmath \hat{B})-8 \hat{J}\left(\mu_{B}^{A} \hat{B}+\mu_{I}^{A} B\right)-8 J\left(\mu_{B}^{A} B+\mu_{I}^{A} \hat{B}\right) \\
& +4\left[\left(\mu_{B}^{A}+\mu_{I}^{A}\right)^{2}-(\hat{\jmath}+\jmath)^{2}\right](\hat{B}+B) \operatorname{coth} \frac{\pi(\hat{B}+B)}{2} \\
& +4\left[\left(\mu_{B}^{A}-\mu_{I}^{A}\right)^{2}-(\hat{\jmath}-\jmath)^{2}\right](\hat{B}-B) \operatorname{coth} \frac{\pi(\hat{B}-B)}{2} .
\end{aligned}
$$

Inserting Eqs. (B.11) and (B.13) into Eq. (3.13) yields the free energy

$$
\begin{aligned}
\Omega= & \frac{2 \kappa M_{\mathrm{KK}}^{2}}{3}\left\{\left[(\jmath+\hat{\jmath})^{2}-\left(\mu_{B}^{A}+\mu_{I}^{A}\right)^{2}\right] \rho_{+}+\left[(\jmath-\hat{\jmath})^{2}-\left(\mu_{B}^{A}-\mu_{I}^{A}\right)^{2}\right] \rho_{-}\right. \\
& -4 \mu_{B}^{V}(\hat{\jmath} \hat{B}+\jmath B)-4 \mu_{I}^{V}(\hat{\jmath} B+\jmath \hat{B}) \\
& \left.+4 \hat{J}\left(\mu_{B}^{A} \hat{B}+\mu_{I}^{A} B\right)+4 J\left(\mu_{B}^{A} B+\mu_{I}^{A} \hat{B}\right)\right\}
\end{aligned}
$$

with $\rho_{ \pm}$defined in Eq. (4.14). For the behavior of $\rho_{ \pm}$for small and large magnetic fields see Table 2. We see that if we allowed for nonzero axial chemical potentials $\mu_{B}^{A}, \mu_{I}^{A}$, the free energy would be unbounded from below in the directions of the sum of left- and right-handed supercurrents $\hat{J}$ and $J$. However, in the physical case of the $\sigma$ phase where $\mu_{B}^{A}=\mu_{I}^{A}=0$ the free energy remains bounded and independent of $\hat{J}$ and $J$. The latter is a manifestation of a residual gauge symmetry ("residual" since we have already employed the gauge $\mathcal{A}_{z}=0$ ), i.e., we can choose a gauge where $\hat{J}=J=0$. This is in accordance with the discussion in Ref. [17], see in particular Eq. (5.23) in this reference. 
Minimization of $\Omega$ with respect to the currents $\hat{\jmath}, \jmath$ yields

$$
\begin{aligned}
& \hat{\jmath}=\frac{\mu_{B}^{V}+\mu_{I}^{V}}{2} \frac{\hat{B}+B}{\rho_{+}}+\frac{\mu_{B}^{V}-\mu_{I}^{V}}{2} \frac{\hat{B}-B}{\rho_{-}}, \\
& \jmath=\frac{\mu_{B}^{V}+\mu_{I}^{V}}{2} \frac{\hat{B}+B}{\rho_{+}}-\frac{\mu_{B}^{V}-\mu_{I}^{V}}{2} \frac{\hat{B}-B}{\rho_{-}},
\end{aligned}
$$

and the minimum of the free energy becomes (with $\mu_{B}^{A}=\mu_{I}^{A}=0$ )

$$
\Omega_{0}=-\frac{2 \kappa M_{\mathrm{KK}}^{2}}{3}\left[\left(\mu_{B}^{V}+\mu_{I}^{V}\right)^{2} \frac{(\hat{B}+B)^{2}}{\rho_{+}}+\left(\mu_{B}^{V}-\mu_{I}^{V}\right)^{2} \frac{(\hat{B}-B)^{2}}{\rho_{-}}\right] .
$$

\section{Solving the equations of motion for nonconstant magnetic fields}

In this appendix we present the general solution to the differential equations (4.8) for the case of a nonconstant isospin magnetic field given in Eq. (4.22). The general expressions given below reduce to the results for the charged pion phase upon inserting the specific boundary conditions from the second row of Table 1. The general boundary conditions considered here are the same as the ones given in Eqs. (B.1).

Then, the solution of (4.8) has the same form as given in Eqs. (B.3) and (B.5), with $\zeta_{ \pm}(z)$ replaced by

$$
\tilde{\zeta}_{ \pm}(z) \equiv e^{\left(\hat{B} \pm \frac{B}{\pi} \arctan z\right) \arctan z} .
$$

To obtain the gauge fields we need

$$
\begin{aligned}
\int d z \frac{\tilde{\zeta}_{+}(z)}{k(z)} & =P_{+}(z), & & \int d z \frac{\tilde{\zeta}_{-}^{-1}(z)}{k(z)}=-P_{-}(z), \\
\int d z \frac{\tilde{\zeta}_{+}^{-1}(z)}{k(z)} & =Q_{+}(z), & & \int d z \frac{\tilde{\zeta}_{-}(z)}{k(z)}=-Q_{-}(z),
\end{aligned}
$$

with $P_{ \pm}, Q_{ \pm}$given in Eqs. (4.25). We shall denote $Q_{ \pm}^{+} \equiv Q_{ \pm}(+\infty), Q_{ \pm}^{-} \equiv Q_{ \pm}(-\infty)$, $P_{ \pm}^{+} \equiv P_{ \pm}(+\infty), P_{ \pm}^{-} \equiv P_{ \pm}(-\infty)$, and use $P_{-}^{ \pm}=P_{+}^{\mp}, Q_{-}^{ \pm}=Q_{+}^{\mp}$. Hence we can express the values of $P_{-}, Q_{-}$at $z= \pm \infty$ through the values of $P_{+}, Q_{+}$at $z=\mp \infty$. Then, after determining the integration constants from the boundary conditions we can write the gauge fields as

$$
\begin{aligned}
\hat{A}_{0}= & 2 \mu_{B}^{V}+\mu_{B}^{A}\left(\tilde{S}_{+}+\tilde{S}_{-}\right)+\jmath\left(\tilde{S}_{+}-\tilde{S}_{-}\right) \\
& +\mu_{I}^{A}\left(\tilde{C}_{+}+\tilde{C}_{-}-\tilde{T}_{+}\right)+\hat{\jmath}\left(\tilde{C}_{+}-\tilde{C}_{-}-\tilde{T}_{-}\right), \\
A_{0}= & 2 \mu_{I}^{V}+\mu_{I}^{A}\left(\tilde{S}_{+}+\tilde{S}_{-}\right)+\hat{\jmath}\left(\tilde{S}_{+}-\tilde{S}_{-}\right) \\
& +\mu_{B}^{A}\left(\tilde{C}_{+}+\tilde{C}_{-}-\tilde{T}_{+}\right)+\jmath\left(\tilde{C}_{+}-\tilde{C}_{-}-\tilde{T}_{-}\right), \\
\hat{A}_{3}= & 2 \hat{J}+\hat{\jmath}\left(\tilde{S}_{+}+\tilde{S}_{-}\right)+\mu_{I}^{A}\left(\tilde{S}_{+}-\tilde{S}_{-}\right) \\
& +\jmath\left(\tilde{C}_{+}+\tilde{C}_{-}-\tilde{T}_{+}\right)+\mu_{B}^{A}\left(\tilde{C}_{+}-\tilde{C}_{-}-\tilde{T}_{-}\right), \\
A_{3}= & 2 J+\jmath\left(\tilde{S}_{+}+\tilde{S}_{-}\right)+\mu_{B}^{A}\left(\tilde{S}_{+}-\tilde{S}_{-}\right) \\
& +\hat{\jmath}\left(\tilde{C}_{+}+\tilde{C}_{-}-\tilde{T}_{+}\right)+\mu_{I}^{A}\left(\tilde{C}_{+}-\tilde{C}_{-}-\tilde{T}_{-}\right),
\end{aligned}
$$


and the field strengths as

$$
\begin{aligned}
& k \hat{F}_{z 0}=\mu_{B}^{A}\left(c_{+}+c_{-}\right)+\jmath\left(c_{+}-c_{-}\right)+\mu_{I}^{A}\left(s_{+}+s_{-}\right)+\hat{\jmath}\left(s_{+}-s_{-}\right), \\
& k F_{z 0}=\mu_{I}^{A}\left(c_{+}+c_{-}\right)+\hat{\jmath}\left(c_{+}-c_{-}\right)+\mu_{B}^{A}\left(s_{+}+s_{-}\right)+\jmath\left(s_{+}-s_{-}\right), \\
& k \hat{F}_{z 3}=\hat{\jmath}\left(c_{+}+c_{-}\right)+\mu_{I}^{A}\left(c_{+}-c_{-}\right)+\jmath\left(s_{+}+s_{-}\right)+\mu_{B}^{A}\left(s_{+}-s_{-}\right), \\
& k F_{z 3}=\jmath\left(c_{+}+c_{-}\right)+\mu_{B}^{A}\left(c_{+}-c_{-}\right)+\hat{\jmath}\left(s_{+}+s_{-}\right)+\mu_{I}^{A}\left(s_{+}-s_{-}\right),
\end{aligned}
$$

where $\tilde{C}_{ \pm}, \tilde{S}_{ \pm}$, and $\tilde{T}_{ \pm}$are defined in Eqs. (4.24), and where

$$
\begin{aligned}
c_{+}(z) \equiv \frac{\tilde{\zeta}_{+}(z)+\tilde{\zeta}_{-}^{-1}(z)}{P_{+}^{+}-P_{+}^{-}}, & c_{-}(z) \equiv \frac{\tilde{\zeta}_{+}^{-1}(z)+\tilde{\zeta}_{-}(z)}{Q_{+}^{+}-Q_{+}^{-}} \\
s_{+}(z) \equiv \frac{\tilde{\zeta}_{+}(z)-\tilde{\zeta}_{-}^{-1}(z)}{P_{+}^{+}-P_{+}^{-}}, & s_{-}(z) \equiv \frac{\tilde{\zeta}_{+}^{-1}(z)-\tilde{\zeta}_{-}(z)}{Q_{+}^{+}-Q_{+}^{-}}
\end{aligned}
$$

(These additional definitions were not necessary in the case of constant magnetic fields, since there the integration of the solution could be expressed in terms of the same functions as the solution itself.)

We now have to check the behavior of the gauge fields $(\overline{C .3})$ under a parity transformation. For the pion phase we have $\mu_{B}^{A}=\mu_{I}^{V}=0$. We have to require $\hat{A}_{0} \rightarrow+\hat{A}_{0}, A_{0} \rightarrow-A_{0}$, $\hat{A}_{3} \rightarrow-\hat{A}_{3}, A_{3} \rightarrow+A_{3}$ (note the additional "twist" for the isospin components originating from the isospin rotation explained in Sec. 4.1). Since $\tilde{C}_{ \pm}(z) \rightarrow+\tilde{C}_{ \pm}(z), \tilde{S}_{ \pm}(z) \rightarrow-\tilde{S}_{ \pm}(z)$, and $\hat{\jmath}, \jmath \rightarrow+\hat{\jmath},+\jmath$ and $\hat{J}, J \rightarrow-\hat{J},-J$ under a parity transformation, we have to require

$$
J=\jmath=0 .
$$

We shall continue with the general solution but have to keep this condition in mind for the final result.

For the free energy we first note that the following combinations are independent of $z$,

$$
c_{+} c_{-}+s_{+} s_{-}=\frac{4}{\left(P_{+}^{+}-P_{+}^{-}\right)\left(Q_{+}^{+}-Q_{+}^{-}\right)}, \quad s_{+} c_{-}+s_{-} c_{+}=0 .
$$

Then, we find

$$
k^{2}\left(-\hat{F}_{z 0}^{2}-F_{z 0}^{2}+\hat{F}_{z 3}^{2}+F_{z 3}^{2}\right)=16 \frac{\left(\hat{\jmath}^{2}+\jmath^{2}\right)-\left[\left(\mu_{B}^{A}\right)^{2}+\left(\mu_{I}^{A}\right)^{2}\right]}{\left(P_{+}^{+}-P_{+}^{-}\right)\left(Q_{+}^{+}-Q_{+}^{-}\right)} .
$$

Next we use that $c_{ \pm}$and $s_{ \pm}$are symmetric and antisymmetric in $z$, respectively, and denote $c_{ \pm}^{+} \equiv c_{ \pm}(\infty)=c_{ \pm}(-\infty), s_{ \pm}^{+} \equiv s_{ \pm}(\infty)=-s_{ \pm}(-\infty)$. Then,

$$
\begin{aligned}
& \left(\hat{A}_{0} k \hat{F}_{z 0}+A_{0} k F_{z 0}-\hat{A}_{3} k \hat{F}_{z 3}-A_{3} k F_{z 3}\right)_{z=-\infty}^{z=\infty} \\
& =4\left(s_{+}^{+}+s_{-}^{+}\right)\left(\mu_{B}^{V} \mu_{I}^{A}+\mu_{I}^{V} \mu_{B}^{A}-\jmath \hat{J}-\hat{\jmath} J\right)+4\left(s_{+}^{+}-s_{-}^{+}\right)\left(\hat{\jmath} \mu_{B}^{V}+\jmath \mu_{I}^{V}-\hat{J} \mu_{B}^{A}-J \mu_{I}^{A}\right) \\
& +4\left(c_{+}^{+}+c_{-}^{+}\right)\left[\left(\mu_{B}^{A}\right)^{2}+\left(\mu_{I}^{A}\right)^{2}-\left(\hat{\jmath}^{2}+\jmath^{2}\right)\right] .
\end{aligned}
$$

Inserting this into the free energy $(3.13)$ yields

$$
\begin{aligned}
\Omega= & \frac{2 \kappa M_{\mathrm{KK}}^{2}}{3}\left\{\left[\hat{\jmath}^{2}+\jmath^{2}-\left(\mu_{B}^{A}\right)^{2}-\left(\mu_{I}^{A}\right)^{2}\right] \rho-2\left(\mu_{B}^{V} \mu_{I}^{A}+\mu_{I}^{V} \mu_{B}^{A}\right) \eta_{+}-2\left(\mu_{B}^{V} \hat{\jmath}+\mu_{I}^{V} \jmath\right) \eta_{-}\right. \\
& \left.+2 \hat{J}\left(\mu_{B}^{A} \eta_{-}+\jmath \eta_{+}\right)+2 J\left(\mu_{I}^{A} \eta_{-}+\hat{\jmath} \eta_{+}\right)\right\},
\end{aligned}
$$


with $\rho$ and $\eta_{ \pm} \equiv s_{+}^{+} \pm s_{-}^{+}$given in Eqs. (4.27); their behavior for small and large magnetic fields can be found in Table 2. As in the case of constant magnetic fields discussed in the previous appendix, see Eq. (B.14), the free energy is unbounded from below without further constraints. This can be seen by computing the matrix of second derivatives $\partial^{2} \Omega /\left(\partial x_{m} \partial x_{n}\right)$

with $x_{m}, x_{n}=\hat{\jmath}, \jmath, \hat{J}, J$. This matrix has eigenvalues $2 \kappa M_{\mathrm{KK}}^{2} / 3\left[\rho \pm\left(\rho^{2}+4 \eta_{+}^{2}\right)^{1 / 2}\right]$, two of which are negative for all magnetic fields. However, we already know from the requirement of a well-defined parity of the gauge fields that $J=\jmath=0$. Then, with $\mu_{B}^{A}=\mu_{I}^{V}=0$, as required for the charged pion condensate, we see that the free energy becomes bounded from below. The only remaining supercurrent with respect to which we need to minimize the free energy is then $\hat{\jmath}$. The sum of left- and right-handed supercurrents, $\hat{J}$, remains undetermined, which is, as mentioned for the case of the sigma phase below Eq. (B.14), a consequence of the residual gauge freedom. We may thus set $\hat{J}=0$.

We can now minimize with respect to $\hat{\jmath}$,

$$
\hat{\jmath}=\mu_{B}^{V} \frac{\eta}{\rho}
$$

and insert this back into the free energy,

$$
\Omega_{0}=-\frac{2 \kappa M_{\mathrm{KK}}^{2}}{3}\left\{\left(\mu_{B}^{V}\right)^{2} \frac{\eta_{-}^{2}}{\rho}+\left(\mu_{I}^{A}\right)^{2} \rho+2 \eta_{+} \mu_{B}^{V} \mu_{I}^{A}\right\} .
$$

\section{Equations of motion and free energy in the chirally restored phase}

Within our approximation of treating the flavor branes as probe branes, the free energies discussed in the main part of the paper are negligible for the finite-temperature phase transition to the chirally restored phase. It is rather the background geometry which is responsible for this phase transition [28, 63]. Therefore, our approach cannot show magnetic-field induced corrections beyond the order of $N_{f} / N_{c}$ to the critical temperature $T_{c}$ for chiral symmetry breaking. This is different when the $\mathrm{D} 8$ and $\overline{\mathrm{D} 8}$ branes are not maximally separated in the extra dimension [94, 95].

In this appendix we simply give the equations of motion and the free energy for the chirally restored phase without discussing the solutions. We do so for the sake of completeness but also because these expressions may be useful to compute possible small corrections to $T_{c}$ of the order of $N_{f} / N_{c}$. One might then speculate whether these corrections persist for smaller and thus more realistic values of $N_{c}$. We leave such a study for the future.

The derivation of the equations of motion and the free energy of the chirally restored phase is analogous to the one for the confined phase given in Sec. 3 and Appendix A. The only difference is the use of the metric (2.9b) instead of (2.9a) and Eq. (2.6) instead of (2.3). We use the same coordinate transformation as in the chirally broken phase, i.e., Eq. (3.1) with $u_{\mathrm{KK}}$ replaced by $u_{T}$ and with $z \in[0, \infty]$. This is not really a simplification in this case but it helps to compare the result to the one for the chirally broken phase. We find for the equations of motion

$$
\partial_{z}\left[k_{3}(z) \partial_{z} \hat{b}\right]=\partial_{z}\left[k_{3}(z) \partial_{z} b\right]=0
$$


and

$$
\begin{aligned}
& \partial_{z}\left[k_{0}(z) \hat{F}_{z 0}\right]=\frac{\alpha M_{\mathrm{KK}} u_{T}^{2}}{(2 \pi T)^{3}}\left[b(z) F_{z 3}+\hat{b}(z) \hat{F}_{z 3}\right], \\
& \partial_{z}\left[k_{0}(z) F_{z 0}\right]=\frac{\alpha M_{\mathrm{KK}} u_{T}^{2}}{(2 \pi T)^{3}}\left[b(z) \hat{F}_{z 3}+\hat{b}(z) F_{z 3}\right], \\
& \partial_{z}\left[k_{3}(z) \hat{F}_{z 3}\right]=\frac{\alpha M_{\mathrm{KK}} u_{T}^{2}}{(2 \pi T)^{3}}\left[b(z) F_{z 0}+\hat{b}(z) \hat{F}_{z 0}\right], \\
& \partial_{z}\left[k_{3}(z) F_{z 3}\right]=\frac{\alpha M_{\mathrm{KK}} u_{T}^{2}}{(2 \pi T)^{3}}\left[b(z) \hat{F}_{z 0}+\hat{b}(z) F_{z 0}\right] .
\end{aligned}
$$

In contrast to the confined phase, there are now two different functions appearing for the temporal and spatial components,

$$
k_{0}(z) \equiv \frac{\left(u_{T}^{3}+u_{T} z^{2}\right)^{3 / 2}}{z u_{T}^{1 / 2}}, \quad k_{3}(z) \equiv z u_{T}^{1 / 2}\left(u_{T}^{3}+u_{T} z^{2}\right)^{1 / 2} .
$$

The free energy becomes

$$
\begin{aligned}
\Omega^{\text {deconf }}= & \Omega_{g}^{\text {deconf }}+\Omega_{b}^{\text {deconf }}+\frac{\kappa(2 \pi T)^{3}}{3 M_{\mathrm{KK}} u_{T}^{2}} \int_{0}^{\infty} d z\left[-k_{0}(z)\left(\hat{F}_{z 0}^{2}+F_{z 0}^{2}\right)+k_{3}(z)\left(\hat{F}_{z 3}^{2}+F_{z 3}^{2}\right)\right] \\
& -\frac{2 \kappa(2 \pi T)^{3}}{3 M_{\mathrm{KK}} u_{T}^{2}}\left[k_{0}(z)\left(\hat{A}_{0} \hat{F}_{z 0}+A_{0} F_{z 0}\right)-k_{3}(z)\left(\hat{A}_{3} \hat{F}_{z 3}+A_{3} F_{z 3}\right)\right]_{z=0}^{z=+\infty},
\end{aligned}
$$

where

$$
\begin{aligned}
& \Omega_{g}^{\text {deconf }} \equiv \frac{32 \kappa(2 \pi T)^{3}}{9\left(2 \pi \alpha^{\prime}\right)^{2} u_{T}^{2} M_{\mathrm{KK}}} \int_{0}^{\infty} d z z u_{T}^{3 / 2}\left(u_{T}^{3}+u_{T} z^{2}\right)^{1 / 6} \\
& \Omega_{b}^{\text {deconf }} \equiv \frac{\kappa(2 \pi T)}{M_{\mathrm{KK}}}\left(\hat{\mathcal{B}}^{2}+\mathcal{B}^{2}\right) \int_{0}^{\infty} d z z u_{T}^{1 / 2}\left(u_{T}^{3}+u_{T} z^{2}\right)^{-5 / 6} .
\end{aligned}
$$

Here we have assumed the magnetic field to be constant in $z, \hat{b}(z)=\hat{\mathcal{B}}, b(z)=\mathcal{B}$, which solves Eq. (D.1). We see that at the critical temperature where $2 \pi T=M_{\mathrm{KK}}$ and thus $u_{T}=u_{\mathrm{KK}}$ the free energy assumes a form very similar to the one in the confined phase. The only differences are then the functions $k_{0}(z)$ and $k_{3}(z)$ (vs. the single function $k(z)$ in the confined phase) and the different integrands in $\Omega_{g}$ and $\Omega_{b}$.

\section{References}

[1] R. C. Duncan and C. Thompson, Formation of very strongly magnetized neutron stars implications for gamma-ray bursts, Astrophys. J. 392 (1992) L9.

[2] D. E. Kharzeev, L. D. McLerran, and H. J. Warringa, The effects of topological charge change in heavy ion collisions: 'Event by event $P$ and CP violation', Nucl. Phys. A803 (2008) 227-253, arXiv:0711.0950.

[3] M. G. Alford, K. Rajagopal, and F. Wilczek, Color-flavor locking and chiral symmetry breaking in high density QCD, Nucl. Phys. B537 (1999) 443-458, hep-ph/9804403. 
[4] V. P. Gusynin, V. A. Miransky, and I. A. Shovkovy, Catalysis of dynamical flavor symmetry breaking by a magnetic field in (2+1)-dimensions, Phys. Rev. Lett. 73 (1994) 3499-3502, hep-ph/9405262.

[5] V. P. Gusynin, V. A. Miransky, and I. A. Shovkovy, Dimensional reduction and catalysis of dynamical symmetry breaking by a magnetic field, Nucl. Phys. B462 (1996) 249-290, hep-ph/9509320.

[6] S. P. Klevansky and R. H. Lemmer, Chiral symmetry restoration in the Nambu-Jona-Lasinio model with a constant electromagnetic field, Phys. Rev. D39 (1989) 3478-3489.

[7] S. P. Klevansky, The Nambu-Jona-Lasinio model of quantum chromodynamics, Rev. Mod. Phys. 64 (1992) 649-708.

[8] N. O. Agasian and I. A. Shushpanov, Gell-Mann-Oakes-Renner relation in a magnetic field at finite temperature, JHEP 10 (2001) 006, hep-ph/0107128.

[9] T. D. Cohen, D. A. McGady, and E. S. Werbos, The chiral condensate in a constant electromagnetic field, Phys. Rev. C76 (2007) 055201, arXiv:0706.3208].

[10] N. O. Agasian and S. M. Fedorov, Quark-hadron phase transition in a magnetic field, Phys. Lett. B663 (2008) 445-449, arXiv:0803.3156.

[11] E. S. Fraga and A. J. Mizher, Chiral transition in a strong magnetic background, Phys. Rev. D78 (2008) 025016, arXiv:0804.1452.

[12] A. J. Mizher and E. S. Fraga, CP violation and chiral symmetry restoration in the hot linear sigma model in a strong magnetic background, arXiv:0810.5162.

[13] E. J. Ferrer, V. de la Incera, and C. Manuel, Magnetic color flavor locking phase in high density QCD, Phys. Rev. Lett. 95 (2005) 152002, hep-ph/0503162.

[14] K. Fukushima and H. J. Warringa, Color superconducting matter in a magnetic field, Phys. Rev. Lett. 100 (2008) 032007, arXiv:0707.3785.

[15] J. L. Noronha and I. A. Shovkovy, Color-flavor locked superconductor in a magnetic field, Phys. Rev. D76 (2007) 105030, arXiv:0708.0307.

[16] D. T. Son and M. A. Stephanov, Axial anomaly and magnetism of nuclear and quark matter, Phys. Rev. D77 (2008) 014021, arXiv:0710.1084.

[17] T. Sakai and S. Sugimoto, Low energy hadron physics in holographic QCD, Prog. Theor. Phys. 113 (2005) 843-882, hep-th/0412141.

[18] T. Sakai and S. Sugimoto, More on a holographic dual of QCD, Prog. Theor. Phys. 114 (2005) 1083-1118, hep-th/0507073.

[19] V. G. Filev, C. V. Johnson, R. C. Rashkov, and K. S. Viswanathan, Flavoured large N gauge theory in an external magnetic field, JHEP 10 (2007) 019, hep-th/0701001].

[20] T. Albash, V. G. Filev, C. V. Johnson, and A. Kundu, Finite Temperature Large N Gauge Theory with Quarks in an External Magnetic Field, JHEP 07 (2008) 080, arXiv:0709.1547.

[21] J. Erdmenger, R. Meyer, and J. P. Shock, AdS/CFT with Flavour in Electric and Magnetic Kalb-Ramond Fields, JHEP 12 (2007) 091, arXiv:0709.1551.

[22] J. M. Maldacena, The large $N$ limit of superconformal field theories and supergravity, Adv. Theor. Math. Phys. 2 (1998) 231-252, hep-th/9711200. 
[23] S. S. Gubser, I. R. Klebanov, and A. M. Polyakov, Gauge theory correlators from non-critical string theory, Phys. Lett. B428 (1998) 105-114, hep-th/9802109.

[24] E. Witten, Anti-de Sitter space and holography, Adv. Theor. Math. Phys. 2 (1998) 253-291, hep-th/9802150.

[25] O. Aharony, S. S. Gubser, J. M. Maldacena, H. Ooguri, and Y. Oz, Large N field theories, string theory and gravity, Phys. Rept. 323 (2000) 183-386, hep-th/9905111.

[26] D. T. Son and M. A. Stephanov, QCD and dimensional deconstruction, Phys. Rev. D69 (2004) 065020, hep-ph/0304182.

[27] J. Erlich, E. Katz, D. T. Son, and M. A. Stephanov, QCD and a holographic model of hadrons, Phys. Rev. Lett. 95 (2005) 261602, hep-ph/0501128.

[28] E. Witten, Anti-de Sitter space, thermal phase transition, and confinement in gauge theories, Adv. Theor. Math. Phys. 2 (1998) 505-532, hep-th/9803131.

[29] A. B. Migdal, Phase Transition in Nuclear Matter and Non-pair Nuclear Forces, Sov. Phys. JETP 36 (1973) 1052.

[30] R. F. Sawyer, Condensed $\pi^{-}$phase in neutron star matter, Phys. Rev. Lett. 29 (1972) $382-385$.

[31] D. J. Scalapino, $\pi^{-}$condensate in dense nuclear matter, Phys. Rev. Lett. 29 (1972) 386-388.

[32] G. Baym, Pion condensation in nuclear and neutron star matter, Phys. Rev. Lett. 30 (1973) $1340-1342$.

[33] A. I. Larkin and Y. N. Ovchinnikov, Inhomogeneous state of superconductors, Sov. Phys. JETP 20 (1965) 762.

[34] P. Fulde and R. A. Ferrell, Superconductivity in a strong spin-exchange field, Phys. Rev. 135 (1964) A550.

[35] M. G. Alford, J. A. Bowers, and K. Rajagopal, Crystalline color superconductivity, Phys. Rev. D63 (2001) 074016, hep-ph/0008208.

[36] O. Schnetz, M. Thies, and K. Urlichs, Phase diagram of the Gross-Neveu model: Exact results and condensed matter precursors, Ann. Phys. 314 (2004) 425-447, hep-th/0402014.

[37] L. He, M. Jin, and P. Zhuang, Pion condensation in baryonic matter: From Sarma phase to LOFF phase, Phys. Rev. D74 (2006) 036005, hep-ph/0604224.

[38] A. Kryjevski, Spontaneous superfluid current generation in the kaon condensed color flavor locked phase at nonzero strange quark mass, Phys. Rev. D77 (2008) 014018, hep-ph/0508180.

[39] T. Schäfer, Meson supercurrent state in high density QCD, Phys. Rev. Lett. 96 (2006) 012305, hep-ph/0508190.

[40] A. Schmitt, Supercurrents in color-superconducting quark matter, Nucl. Phys. A820 (2009) 49c-56c, arXiv:0810.4243.

[41] K. Nawa, H. Suganuma, and T. Kojo, Baryons in Holographic QCD, Phys. Rev. D75 (2007) 086003, hep-th/0612187.

[42] H. Hata, T. Sakai, S. Sugimoto, and S. Yamato, Baryons from instantons in holographic $Q C D$, hep-th/0701280. 
[43] K. Hashimoto, T. Sakai, and S. Sugimoto, Holographic Baryons : Static Properties and Form Factors from Gauge/String Duality, arXiv:0806.3122.

[44] K. Nawa, H. Suganuma, and T. Kojo, Brane-induced Skyrmion on $S^{3}$ : baryonic matter in holographic $Q C D$, arXiv:0810.1005.

[45] S. Seki and J. Sonnenschein, Comments on Baryons in Holographic QCD, JHEP 01 (2009) 053, arXiv:0810.1633.

[46] M. Rozali, H.-H. Shieh, M. Van Raamsdonk, and J. Wu, Cold Nuclear Matter In Holographic QCD, JHEP 01 (2008) 053, arXiv:0708.1322].

[47] K.-Y. Kim, S.-J. Sin, and I. Zahed, The Chiral Model of Sakai-Sugimoto at Finite Baryon Density, JHEP 01 (2008) 002, arXiv:0708.1469.

[48] K.-Y. Kim, S.-J. Sin, and I. Zahed, Dense Holographic QCD in the Wigner-Seitz Approximation, JHEP 09 (2008) 001, arXiv:0712.1582.

[49] O. Bergman, G. Lifschytz, and M. Lippert, Magnetic properties of dense holographic QCD, arXiv:0806.0366v3.

[50] E. G. Thompson and D. T. Son, Magnetized baryonic matter in holographic QCD, Phys. Rev. D78 (2008) 066007, arXiv:0806.0367].

[51] S. A. Hartnoll, C. P. Herzog, and G. T. Horowitz, Building a Holographic Superconductor, Phys. Rev. Lett. 101 (2008) 031601, arXiv:0803.3295.

[52] S. S. Gubser and S. S. Pufu, The gravity dual of a p-wave superconductor, JHEP 11 (2008) 033, arXiv:0805.2960.

[53] P. Basu, A. Mukherjee, and H.-H. Shieh, Supercurrent: Vector Hair for an AdS Black Hole, arXiv:0809.4494.

[54] C. P. Herzog, P. K. Kovtun, and D. T. Son, Holographic model of superfluidity, arXiv:0809.4870.

[55] M. Ammon, J. Erdmenger, M. Kaminski, and P. Kerner, Superconductivity from gauge/gravity duality with flavor, arXiv:0810.2316.

[56] P. Basu, J. He, A. Mukherjee, and H.-H. Shieh, Superconductivity from D3/D\%: Holographic Pion Superfluid, arXiv:0810.3970.

[57] S. A. Hartnoll, C. P. Herzog, and G. T. Horowitz, Holographic Superconductors, JHEP 12 (2008) 015, arXiv:0810.1563.

[58] M. Kruczenski, D. Mateos, R. C. Myers, and D. J. Winters, Towards a holographic dual of large- $N_{c} Q C D, J H E P 05$ (2004) 041, hep-th/0311270.

[59] L. McLerran and R. D. Pisarski, Phases of Cold, Dense Quarks at Large $N_{c}$, Nucl. Phys. A796 (2007) 83-100, arXiv:0706.2191.

[60] O. Aharony and D. Kutasov, Holographic Duals of Long Open Strings, Phys. Rev. D78 (2008) 026005, arXiv:0803.3547.

[61] P. C. Argyres, M. Edalati, R. G. Leigh, and J. F. Vazquez-Poritz, Open Wilson Lines and Chiral Condensates in Thermal Holographic QCD, Phys. Rev. D79 (2009) 045022, arXiv:0811.4617. 
[62] O. Aharony, J. Sonnenschein, and S. Yankielowicz, A holographic model of deconfinement and chiral symmetry restoration, Annals Phys. 322 (2007) 1420-1443, hep-th/0604161.

[63] N. Horigome and Y. Tanii, Holographic chiral phase transition with chemical potential, JHEP 01 (2007) 072, hep-th/0608198.

[64] E. Antonyan, J. A. Harvey, S. Jensen, and D. Kutasov, NJL and QCD from string theory, hep-th/0604017.

[65] A. Parnachev, Holographic QCD with Isospin Chemical Potential, JHEP 02 (2008) 062, arXiv:0708.3170.

[66] O. Aharony, K. Peeters, J. Sonnenschein, and M. Zamaklar, Rho meson condensation at finite isospin chemical potential in a holographic model for QCD, JHEP 02 (2008) 071, arXiv:0709.3948.

[67] D. Vollhardt and P. Wölfle, The Superfluid Phases of Helium 3. Taylor \& Francis, London, 1990.

[68] D. T. Son, Low-energy quantum effective action for relativistic superfluids, hep-ph/0204199.

[69] H. Hata, M. Murata, and S. Yamato, Chiral currents and static properties of nucleons in holographic QCD, Phys. Rev. D78 (2008) 086006, arXiv:0803.0180.

[70] A. Karch, A. O'Bannon, and K. Skenderis, Holographic renormalization of probe D-branes in AdS/CFT, JHEP 04 (2006) 015, hep-th/0512125.

[71] T. Wiseman and B. Withers, Holographic renormalization for coincident Dp-branes, JHEP 10 (2008) 037, arXiv:0807.0755.

[72] I. Kanitscheider, K. Skenderis, and M. Taylor, Precision holography for non-conformal branes, JHEP 09 (2008) 094, arXiv:0807.3324.

[73] A. Armoni, Witten-Veneziano from Green-Schwarz, JHEP 06 (2004) 019, hep-th/0404248.

[74] J. L. F. Barbon, C. Hoyos-Badajoz, D. Mateos, and R. C. Myers, The holographic life of the $\eta^{\prime}$, JHEP 10 (2004) 029, hep-th/0404260].

[75] O. Bergman and G. Lifschytz, Holographic U(1) A and string creation, JHEP 04 (2007) 043, hep-th/0612289.

[76] A. Parnachev and A. R. Zhitnitsky, Phase Transitions, $\theta$ Behavior and Instantons in $Q C D$ and its Holographic Model, arXiv:0806.1736.

[77] B. Klein, D. Toublan, and J. J. M. Verbaarschot, The QCD phase diagram at nonzero temperature, baryon and isospin chemical potentials in random matrix theory, Phys. Rev. D68 (2003) 014009, hep-ph/0301143].

[78] E. Witten, Theta dependence in the large $N$ limit of four-dimensional gauge theories, Phys. Rev. Lett. 81 (1998) 2862-2865, hep-th/9807109.

[79] D. Boer and J. K. Boomsma, Spontaneous CP-violation in the strong interaction at $\theta=\pi$, Phys. Rev. D78 (2008) 054027, arXiv:0806.1669.

[80] M. G. Alford, A. Schmitt, K. Rajagopal, and T. Schäfer, Color superconductivity in dense quark matter, Rev. Mod. Phys. 80 (2008) 1455-1515, arXiv:0709.4635.

[81] D. V. Deryagin, D. Y. Grigoriev, and V. A. Rubakov, Standing wave ground state in high density, zero temperature QCD at large $N_{c}$, Int. J. Mod. Phys. A7 (1992) 659-681. 
[82] E. Shuster and D. T. Son, On finite-density QCD at large $N_{c}$, Nucl. Phys. B573 (2000) 434-446, hep-ph/9905448.

[83] L. Y. Glozman and R. F. Wagenbrunn, Chirally symmetric but confining dense and cold matter, Phys. Rev. D77 (2008) 054027, arXiv:0709.3080.

[84] A. Fetter and J. Walecka, Quantum theory of many-particle systems. McGraw-Hill, New York, 1971.

[85] R. McNees, R. C. Myers, and A. Sinha, On quark masses in holographic QCD, JHEP 11 (2008) 056, arXiv:0807.5127.

[86] L.-y. He, M. Jin, and P.-f. Zhuang, Pion superfluidity and meson properties at finite isospin density, Phys. Rev. D71 (2005) 116001, hep-ph/0503272.

[87] B. J. Harrington and H. K. Shepard, Superconducting properties of pion condensates, Phys. Rev. D16 (1977) 3437.

[88] B. J. Harrington and H. K. Shepard, Superconducting properties of pion condensates. II, Phys. Rev. D19 (1979) 1713.

[89] N. Y. Anisimov and D. N. Voskresensky, Superconductivity of pion condensate, Sov. J. Nucl. Phys. 30 (1979) 612-615.

[90] A. B. Migdal, E. E. Saperstein, M. A. Troitsky, and D. N. Voskresensky, Pion degrees of freedom in nuclear matter, Phys. Rept. 192 (1990) 179-437.

[91] H. Abuki, R. Anglani, R. Gatto, M. Pellicoro, and M. Ruggieri, The fate of pion condensation in quark matter: from the chiral to the real world, Phys. Rev. D79 (2009) 034032, arXiv:0809.2658.

[92] J. O. Andersen and L. Kyllingstad, Pion condensation at finite temperature and density: The role of charge neutrality, hep-ph/0701033.

[93] K. Fukushima, D. E. Kharzeev, and H. J. Warringa, The Chiral Magnetic Effect, Phys. Rev. D78 (2008) 074033, arXiv:0808.3382.

[94] O. Bergman, G. Lifschytz, and M. Lippert, Response of Holographic QCD to Electric and Magnetic Fields, JHEP 05 (2008) 007, arXiv:0802.3720.

[95] C. V. Johnson and A. Kundu, External Fields and Chiral Symmetry Breaking in the SakaiSugimoto Model, JHEP 12 (2008) 053, arXiv:0803.0038. 